\title{
On the Development of Solutions of Tricomi's Differential Equation in the Vicinity of the Origin
}

\section{GOTTFRIED GUDERLEY}

\author{
Communicated by C. C. LIN
}

\section{Introduction}

The hodograph treatment of plane transonic potential flows leads to TrICom's equation (Ref. 1)

$$
\psi_{\eta \eta}-\eta \psi_{\theta \theta}=0
$$

Here $\theta$ is the angle of the velocity vector with a fixed axis, $\eta$ is proportional to the deviation of the absolute value of the velocity from the sonic velocity, and $\psi$ is the stream function. The physical significance of these quantities is without importance in the present paper.

In the treatment of flow patterns with a free stream Mach number close to one (Ref. 2 and Ref. 3), one tries to express certain solutions of Eq. (i.1) by a superposition of particular solutions of the form

$$
\psi=|\eta|^{n} \cdot f(\zeta)
$$

where

$$
\zeta=\frac{9}{4} \frac{\theta^{2}}{\eta^{3}}
$$

The values of $n$ depend upon the boundary conditions of the problem. Physical considerations require that $\psi$ and its derivatives be finite along the characteristics $\theta= \pm \frac{2}{3} \eta^{\frac{3}{2}}$. There exists a family of particular solutions which fulfill this requirement; it will be denoted as the first family of particular solutions. Unfortunately it is not clear which class of functions can be represented by a superposition of these expressions. 
This difficulty can be overcome by modifying the boundary conditions in a somewhat artificial manner. A second family of particular solutions is determined by an eigenvalue problem, and the question of completeness is settled immediately. But here the individual particular solutions do not display the expected behavior; some of the functions are not continuous in the vicinity of the characteristics mentioned above, and they are also singular at the origin.

The present paper will show, by means of the theory of analytic functions, how the two representations are related to each other. In this process the limits of applicability of the first system will be determined. For a special example in which this technique has been used, see Ref. 4 .

Since in the course of the coming investigations we are concerned with numerous details which may obscure the general trend of thought, a general survey of the contents of the paper may be worthwhile.

First, detailed formulæ for the particular solutions mentioned above are given, and also their asymptotic representations for large values of $n$ are shown. When boundary conditions are imposed, one obtains the two systems of particular solutions to which we referred previously. Inserting here the asymptotic expressions for large values of $n$, one finds the shapes of the regions of convergence for a superposition of such particular solutions.

Next, a formula is derived which represents an arbitrary function of one variable in terms of the eigenfunctions of the second system of particular solutions.

After these preparations, the problem of representing a solution of TRICOMI's equation by a superposition of these particular solutions is attacked. It is seen easily that this problem is equivalent to an inhomogeneous boundary value problem which requires the determination of a solution $\psi$ of TrIComI's equation for which, at a given curve of the $\eta, \theta$-plane, the jump of $\psi$ and of a suitable outer derivative is prescribed.

The solution of this inhomogeneous boundary value problem is first found using the second system of particular solutions.

Then by means of the theory of functions of a complex variable this result is transformed in such a manner that it appears as a superposition of the particular solutions of the first family.

\section{Particular Solutions}

Eq. (i.2) if inserted into Tricomr's equation (i.1) yields the following hypergeometric differential equation

$$
f^{\prime \prime} \zeta(\zeta-1)+f^{\prime}\left[\left(\frac{4}{3}-\frac{2}{3} n\right) \zeta-\frac{1}{2}\right]+\frac{n(n-1)}{g} \cdot f=0
$$

This equation and its solutions are discussed in Ref. 3. In Ref. 2 Eq. (i.2) is rewritten as

$$
\psi=\rho^{n / 3} \cdot G(\xi)
$$


where

$$
\begin{gathered}
\rho=-\eta^{3}+\left(\frac{3}{2} \theta\right)^{2}=\eta^{3}(\zeta-1) \\
\xi=\frac{\eta}{\left(\frac{3}{2} \theta\right)^{\frac{2}{3}}}=\zeta^{-\frac{1}{3}}
\end{gathered}
$$

The $\rho \xi$-system is shown in Figure 1.

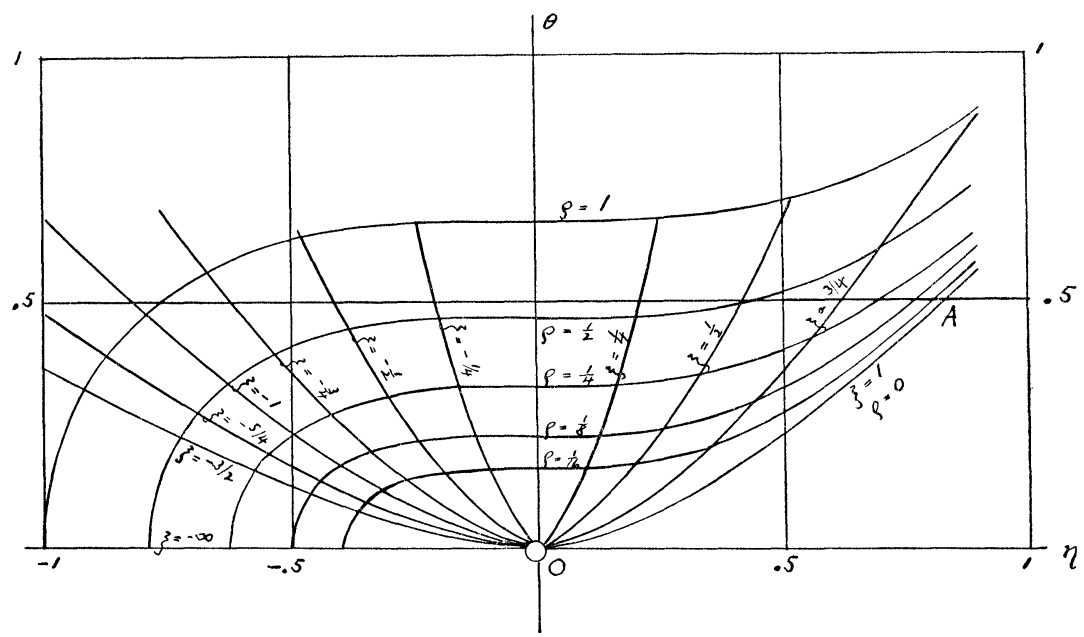

FIG. 1.

Comparing Eqs. (i.2) and (1.2), one finds the following relation between $f$ and $G$ :

$$
f(\zeta)=|(1-\zeta)|^{n / 3} \cdot G\left(\zeta^{-\frac{1}{3}}\right)
$$

After a transformation to the variables $\rho$ and $\xi$, TRICOMI's equation (i.1) assumes the form

$$
\frac{\left(1-\xi^{3}\right)^{2}}{\xi_{-}} \cdot \psi_{\xi \xi}-\frac{5}{2} \xi\left(1-\xi^{3}\right) \psi_{\xi}=9 \rho^{2} \psi_{\rho \rho}+\frac{21}{2} \rho \psi_{\rho}
$$

Occasionally the following form is more practical:

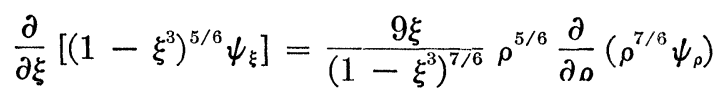

Inserting here Eq. (1.2), one obtains the following differential equation for $G$ :

$$
\frac{d}{d \xi}\left[\left(1-\xi^{3}\right)^{5 / 6} \cdot G^{\prime}\right]+\left(-\lambda+\frac{1}{16}\right) \frac{\xi}{\left(1-\xi^{3}\right)^{7 / 6}} \cdot G=0
$$


where

$$
\lambda=\left(n+\frac{1}{4}\right)^{2}
$$

The range of values $\xi$ in which we are interested extends from $-\infty$ to $1 ; \rho$ varies between 0 and $\infty$. Equation (1.7) is the differential equation of a classical eigenvalue problem except for the change of sign of the coefficient of $G$ occurring at $\xi=0$. Thus, if suitable boundary conditions are imposed, a system of particular solutions is defined for which completeness can be established easily.

Any solution $\psi$ can be split into its symmetric and its antisymmetric part with respect to the axis $\theta=0(\xi=-\infty)$. These parts satisfy equation (i.1) separately. For simplicity only antisymmetric solutions of TrIComi's equation will be considered here.

For the antisymmetric solutions one has along $\xi=-\infty$ the condition $\psi=0$, and consequently $G=0$. We define $G$ by the further requirement that its development at $\xi=-\infty$ starts with the term $(-\xi)^{-\frac{3}{2}}$. For simplicity let

$$
\mu=\frac{\sqrt{\lambda}}{3}=\frac{n}{3}+\frac{1}{12}
$$

The function $G$ just defined is a function of the two variables $\xi$ and $\mu$; this will be expressed by the notation $G(\xi, \mu)$. Since the differential equation (1.7) contains only $\mu^{2}$, we have $G(\xi, \mu)=G(\xi,-\mu)$. Representations of the function $G$ are found in Eqs. (18) of Ref. 2. We have

$$
\begin{gathered}
G=\left(1-\xi^{3}\right)^{-\frac{1}{2}} F\left(-\mu+\frac{7}{12}, \mu+\frac{7}{12}, \frac{3}{2},\left(1-\xi^{3}\right)^{-1}\right) \\
\text { valid from } \xi=-\infty \text { to } \xi=0 \\
G=\frac{\frac{3}{2} \Gamma\left(\frac{4}{3}\right) \cdot \Gamma\left(\frac{1}{2}\right)}{\Gamma\left(\mu+\frac{11}{12}\right) \cdot \Gamma\left(-\mu+\frac{11}{12}\right)} \cdot F\left(-\mu+\frac{1}{12}, \mu+\frac{1}{12}, \frac{2}{3}, \frac{\xi^{3}}{\xi^{3}-1}\right) \\
-\frac{\frac{3}{2} \Gamma\left(\frac{2}{3}\right) \cdot \Gamma\left(\frac{1}{2}\right)}{\Gamma\left(\mu+\frac{7}{12}\right) \cdot \Gamma\left(-\mu+\frac{7}{12}\right)} \cdot \frac{\xi}{\left.\xi^{3}-1\right)^{\frac{1}{3}}} \cdot F\left(-\mu+\frac{5}{12}, \mu+\frac{5}{12}, \frac{4}{3}, \frac{\xi^{3}}{\xi^{3}-1}\right) \\
\quad \text { valid from } \xi=-\infty \text { to } \xi=2^{-\frac{1}{2}} \\
+\frac{\frac{3}{2} \Gamma\left(\frac{4}{3}\right) \cdot \Gamma\left(\frac{1}{2}\right)}{\Gamma\left(\mu+\frac{11}{12}\right) \cdot \Gamma\left(-\mu+\frac{11}{12}\right)}\left(1-\xi^{3}\right)^{1 / 12-\mu} \cdot F\left(-\mu+\frac{1}{12},-\mu+\frac{7}{12}, \frac{2}{3}, \xi^{3}\right) \\
+\frac{\frac{3}{2} \Gamma\left(\frac{2}{3}\right) \cdot \Gamma\left(\frac{1}{2}\right)}{\Gamma\left(\mu+\frac{7}{12}\right) \cdot \Gamma\left(-\mu+\frac{7}{12}\right)}\left(1-\xi^{3}\right)^{1 / 12-\mu} \cdot \xi \cdot F\left(-\mu+\frac{5}{12},-\mu+\frac{11}{12}, \frac{4}{3}, \xi^{3}\right) \\
\text { valid from } \xi=-1 \text { to } \xi=1
\end{gathered}
$$




$$
\begin{gathered}
G=\Gamma\left(\frac{1}{2}\right)\left\{\frac{\Gamma(2 \mu) \sin \left[\pi\left(\mu+\frac{1}{4}\right)\right]}{\Gamma\left(\mu+\frac{11}{12}\right) \cdot \Gamma\left(\mu+\frac{7}{12}\right)}\right. \\
\cdot\left(1-\xi^{3}\right)^{1 / 12-\mu} F\left(-\mu+\frac{1}{12},-\mu+\frac{7}{12},-2 \mu+1,\left(1-\xi^{3}\right)\right) \quad(1.10 \mathrm{~d}) \\
+\frac{\Gamma(-2 \mu) \sin \left[\pi\left(-\mu+\frac{1}{4}\right)\right]}{\Gamma\left(-\mu+\frac{11}{12}\right) \cdot \Gamma\left(-\mu+\frac{7}{12}\right)} \\
\left.\cdot\left(1-\xi^{3}\right)^{1 / 12+\mu} F\left(\mu+\frac{1}{12}, \mu+\frac{7}{12}, 2 \mu+1,\left(1-\xi^{3}\right)\right)\right\}
\end{gathered}
$$

Later, approximate expressions for the function $G$ for large values of $\mu$ will be needed. Here, only the results of the somewhat cumbersome derivations (see e.g. Ref. 5) may be given.

Let

$$
\xi^{\prime}=\frac{1}{2} \log \frac{1+\xi^{\frac{3}{2}}}{1-\xi^{\frac{3}{2}}} \text { for } \xi \geqq 0
$$

and

$$
\xi^{\prime}=t g^{-1}\left[(-\xi)^{\frac{3}{2}}\right] \quad \text { for } \quad \xi \leqq 0
$$

Here the branch of the functions will be used which gives $\xi^{\prime}=0$ for $\xi=0$. Then, for large positive values of $\mu$ and for $\xi \leqq 0$

$$
\begin{array}{r}
G \sim \pi^{1 / 2}(3 \mu)^{-1 / 2}\left|\frac{1-\xi^{3}}{\xi^{3}}\right|^{1 / 12} \cdot\left(\xi^{\prime}\right)^{1 / 2}\left[-\cos \left[\pi\left(\mu+\frac{1}{12}\right)\right] J_{1 / 3}\left(2 \mu \xi^{\prime}\right)\right. \\
\left.+\cos \left[\pi\left(\mu-\frac{5}{12}\right)\right] J_{-1 / 3}\left(2 \mu \xi^{\prime}\right)\right]
\end{array}
$$

for large positive values of $\mu$ and for $0 \leqq \xi \leqq 1$

$$
\begin{aligned}
G \sim \pi^{1 / 2}(3 \mu)^{-1 / 2}\left|\frac{1-\xi^{3}}{\xi^{3}}\right|^{1 / 12}\left(\xi^{\prime}\right)^{1 / 2}\left[\cos \left[\pi\left(\mu-\frac{1}{12}\right)\right] e^{-i \pi / 6} J_{1 / 3}\left(2 \mu i \xi^{\prime}\right)\right. \\
+\cos \left[\pi\left(\mu-\frac{5}{12}\right)\right] e^{i \pi / 6} J_{-1 / 3}\left(2 \mu i \xi^{\prime}\right)
\end{aligned}
$$

The following representations are not valid for $\xi=0$ :

$$
\begin{aligned}
G \sim \frac{1}{2} \mu^{-1}\left|\frac{1-\xi^{3}}{\xi^{3}}\right|^{1 / 12} \sin \left[2 \mu\left(\frac{\pi}{2}-\xi^{\prime}\right)\right] & \text { for } \xi<0 \\
G \sim \frac{1}{2} \mu^{-1}\left|\frac{1-\xi^{3}}{\xi^{3}}\right|^{1 / 12}\left\{\left(\frac{1+\xi^{3 / 2}}{1-\xi^{3 / 2}}\right)^{\mu} \sin \left[\pi\left(\mu+\frac{1}{4}\right)\right]\right. & \text { for } \xi>0 \\
& \left.+\frac{1}{2}\left(\frac{1+\xi^{3 / 2}}{1-\xi^{3 / 2}}\right)^{-\mu} \sin \left[\pi\left(-\mu+\frac{1}{4}\right)\right]\right\}
\end{aligned}
$$


The second term in the braces of the last equation is asymptotically small in comparison with the first term; however, it is important if the coefficient of the first term vanishes.

Furthermore, asymptotic representations for $G$ for large imaginary values of $\mu$ are needed. The differential equation (1.7) for $G$ shows that $G$ is real. Here one has the following representations:

$$
\begin{aligned}
G=\frac{1}{2} \pi^{1 / 2}|3 \mu|^{-1 / 2}\left|\frac{1-\xi^{3}}{\xi^{3}}\right|^{1 / 12}\left|\xi^{\prime}\right|^{1 / 2} e^{\pi|\mu|}\left\{e^{-i \pi / 6} J_{1 / 3}\left(2|\mu| i \xi^{\prime}\right)\right. \\
\\
\left.\quad+e^{i \pi / 6} J_{-1 / 3}\left(2|\mu| i \xi^{\prime}\right)\right\} \text { for }-M<\xi<0
\end{aligned}
$$

where $M$ is any positive number,

$$
\begin{array}{lr}
G=\frac{1}{2} \pi^{1 / 2}|3 \mu|^{-1 / 2}\left|\frac{1-\xi^{3}}{\xi^{3}}\right|^{1 / 12}\left|\xi^{\prime}\right|^{1 / 2} e^{\pi|\mu|}\left\{J_{1 / 3}\left(2|\mu| \xi^{\prime}\right)+J_{-1 / 3}\left(2|\mu| \xi^{\prime}\right)\right\} & \text { for } 0 \leqq \xi \leqq 1 \\
G=\frac{1}{2}|\mu|^{-1}\left|\frac{1-\xi^{3}}{\xi^{3}}\right|^{1 / 12} \sinh \left[2|\mu|\left(\frac{\pi}{2}-\xi^{\prime}\right)\right] & \text { for } \quad \xi<0 \\
G=\frac{1}{2}|\mu|^{-1}\left|\frac{1-\xi^{3}}{\xi^{3}}\right|^{1 / 12} e^{\pi|\mu|} \cos \left[2|\mu|\left(\xi^{\prime}-\frac{\pi}{4}\right)\right. & \text { for } 0<\xi<1
\end{array}
$$

If $|\mu|$ is large and if the real part of $\mu$ is non-negative, one has

$$
\begin{aligned}
& G=\mu^{-1}\left|\frac{1-\xi^{3}}{\xi^{3}}\right|^{1 / 12} \sin \left[2 \mu\left(\frac{\pi}{2}-\xi^{\prime}\right)\right] O(1) \quad \xi<0 \\
& G=\mu^{-1}\left|\frac{1-\xi^{3}}{\xi^{3}}\right|^{1 / 12}\left(\frac{1+\xi^{3 / 2}}{1-\xi^{3 / 2}}\right)^{\mu} e^{\pi \operatorname{Im} \mu} O(1) \quad \xi>0
\end{aligned}
$$

Finally, asymptotic representations are needed for the following particular solutions of the equation for $G$ which occur in Eq. (1.10d)

$$
G_{1}(\xi, \mu)=\left(1-\xi^{3}\right)^{1 / 12+\mu} F\left(\mu+\frac{1}{12}, \mu+\frac{7}{12}, 2 \mu+1,\left(1-\xi^{3}\right)\right)
$$

Note that in contrast to Eq. (1.9)

$$
G_{1}(\xi, \mu) \neq G_{1}(\xi,-\mu)
$$

Here one has

$$
G_{1}(\xi, \mu) \sim\left|\frac{1-\xi^{3}}{\xi^{3}}\right|^{1 / 12}\left(\frac{1-\xi^{3 / 2}}{1+\xi^{3 / 2}}\right)^{\mu} \cdot 2^{2 \mu}
$$

valid for $\left(1-\xi^{3}\right)<1 ;|\mu| \gg 1$ except for a neighborhood of the negative integral and half integral values of $\mu$. That such values must be excluded is obvious from the third parameter $2 \mu+1$ of the hypergeometric function in (1.17). 


\section{Systems of Particular Solutions}

Frequently physical or mathematical considerations indicate that the function $\psi$ and all its derivatives are finite along the characteristic $\theta=\frac{2}{3} \eta^{\frac{3}{2}}$ (which coincides with the line $\xi=1$ ), with the possible exception of the origin of the $\eta, \theta-$ system. By this requirement, a first family of particular solutions is determined.

In the vicinity of $\xi=1$ the hypergeometric series occurring in Eq. (1.10d) can be set equal to 1 , and one obtains as representation of the particular solutions (1.2)

$$
\begin{aligned}
\psi=\rho^{-1 / 12+\mu} \Gamma\left(\frac{1}{2}\right)\left\{\frac{\Gamma(2 \mu) \sin \left[\pi\left(\mu+\frac{1}{4}\right)\right]}{\Gamma\left(\mu+\frac{11}{12}\right) \Gamma\left(\mu+\frac{7}{12}\right)}\left(1-\xi^{3}\right)^{1 / 12-\mu}\right. & \\
& \left.+\frac{\Gamma(-2 \mu) \sin \left[\pi\left(-\mu+\frac{1}{4}\right)\right]}{\Gamma\left(-\mu+\frac{11}{12}\right) \Gamma\left(-\mu+\frac{7}{12}\right)}\left(1-\xi^{3}\right)^{1 / 12+\mu}\right\}
\end{aligned}
$$

The behavior of this expression is displayed more distinctly if one expresses $\rho$ by $\eta$ and $\xi$ according to Eqs. (1.3a) and (1.3b)

$\psi=\left(\frac{\eta}{\xi}\right)^{-\frac{1}{4}+\mu} \Gamma\left(\frac{1}{2}\right)\left\{\frac{\Gamma(2 \mu) \sin \left[\pi\left(\mu+\frac{1}{4}\right)\right]}{\Gamma\left(\mu+\frac{1}{12}\right) \Gamma\left(\mu+\frac{7}{12}\right)}+\frac{\Gamma(-2 \mu) \sin \left[\pi\left(-\mu+\frac{1}{4}\right)\right]}{\Gamma\left(-\mu+\frac{11}{12}\right) \Gamma\left(-\mu+\frac{7}{12}\right)}\left(1-\xi^{3}\right)^{2 \mu}\right\}$

Now, the condition that $\psi$ and its derivatives be finite for $\xi=1$ requires that the coefficient of the second term be zero or that the exponent of $\left(1-\xi^{3}\right)$ in this term be an integer. The second possibility does not yield the desired result: it occurs if the exponents of the hypergeometric equation at the point $\xi=1$ differ by an integer; then the hypergeometric series are not directly applicable, and the solution will contain a logarithmic term.

The coefficient of the second term in the last equation vanishes if

$$
\mu=\frac{1}{4}+\frac{h}{3}
$$

or

$$
\mu=-\frac{3}{4}-h
$$

where $h$ is a non-negative integer. One notices that the positive values of $\mu$ are evenly spaced at intervals of $\frac{1}{3}$; the negative ones are evenly spaced at intervals of 1 .

This first system of particular solutions is determined by the requirement that $\psi$ and all of its derivatives be finite at $\xi=1$. This property is suggested by the nature of the functions $\psi$ which are to be represented by a superposition of these particular solutions. Indeed there are problems, the solutions of which appear directly as a superposition of these particular solutions [Ref. 6 and 7]. The question is, under what conditions such a representation would be valid in a more general case. 
The value of such a representation lies in the fact that along each line $\xi=$ const. the function $\psi$ is then expressed as a power series in $\rho$. This gives a clear picture of the predominant terms in the vicinity of the point $\rho=0$. On the other hand, this form of the representation shows also its limitations. Only a rather restricted class of functions can be represented by power series; e.g., it is not possible that singularities in any of the derivatives of $\psi$ occur within the region of validity of this series. However, singularities at least in the higher derivatives propagating along characteristics will be encountered in the supersonic region unless very severe restrictions, which are not always physically justified, are imposed on the boundary values.

The second system of particular solutions is obtained by imposing the condition $G=0$ for $\xi=c_{2}$, where $c_{2}$ is a constant. Subsequently, a limiting process $c_{2} \rightarrow 1$ will be carried out.

Assume first that $c_{2} \neq 1$. Then we have an eigenvalue problem for the functions $G$. With the preliminary notation $G_{h}$ and $G_{k}$ for two different eigenfunctions, one finds from Eq. (1.7) the following relations of orthogonality:

$$
\begin{array}{ll}
\int_{-\infty}^{c_{2}} \frac{\xi}{\left(1-\xi^{3}\right)^{7 / 6}} G_{h} G_{k} d \xi=0 & h \neq k \\
\int_{-\infty}^{c_{2}}\left(1-\xi^{3}\right)^{5 / 6} \frac{d G_{h}}{d \xi} \frac{d G_{k}}{d \xi}=0 & h \neq k
\end{array}
$$

It follows from Eq. (2.4b) that all eigenvalues $\lambda$ are real. Since the coefficient of $G$ in Eq. (1.7) changes its sign, there exist an infinite number of positive and an infinite number of negative eigenvalues. The completeness of the system of eigenvalues can be shown by means of HILBERT's theory of the polar integral equation. (See e.g., Ref. 8).

The eigenvalues may be arranged according to their magnitude and denoted by

$$
\cdots \lambda_{-h} \cdots \lambda_{-3}, \lambda_{-2}, \lambda_{-1}, \lambda_{1}, \lambda_{2}, \lambda_{3} \cdots \lambda_{h} \cdots
$$

where the negative subscripts refer to the negative, the positive subscripts to the positive eigenvalues. The functions $G$ will be denoted accordingly.

Then one has the following system of particular solutions formed with these eigenfunctions:

$$
\begin{aligned}
\psi_{\mathrm{I} h} & =\rho^{-1 / 12+\left(\sqrt{\lambda_{h}} / 3\right)} G_{h}(\xi) \\
\psi_{\mathrm{II} h} & =\rho^{-1 / 12-\left(\sqrt{\lambda_{h}} / 3\right)} G_{h}(\xi) \\
\psi_{\mathrm{III} h} & =\rho^{-1 / 12} \cos \left(\frac{\sqrt{\left|\lambda_{-h}\right|}}{3} \log \rho\right) G_{-h}(\xi) \\
\psi_{\mathrm{IV} h} & =\rho^{-1 / 12} \sin \left(\frac{\sqrt{\left|\lambda_{-h}\right|}}{3} \log \rho\right) G_{-h}(\xi)
\end{aligned}
$$

Here the $h$ 's are positive integers. 
Consider a function $\psi$ which satisfies TRIComI's differential equation in a region given by $-\infty<\xi<c_{2}$ and $\rho_{1}<\rho<\rho_{2}, \rho_{1}$ and $\rho_{2}$ being constants, and which assumes the value $\psi=0$ for $\xi=-\infty$ and $\xi=c_{2}$. It is shown in Ref. 2, Appendix 1, that such a function can be represented by a superposition of the expressions (2.5).

The eigenvalues $\lambda$ for the limiting case $c_{2} \rightarrow 1$ will be determined in Section 4

\section{Regions of Convergence}

To determine the boundaries of a region in which an infinite series formed with the particular solutions discussed in the previous chapter would converge, we use the representations for large values of $\mu$ derived in Section 1 .

a. Real values of $\mu, \xi<0$

Using Eq. (1.13a), one finds the following approximate representation for a particular solution

$$
\psi=\rho^{-1 / 12+\mu} G(\xi, \mu) \sim \rho^{-1 / 12+\mu}\left|\frac{1-\xi^{3}}{\xi^{3}}\right|^{1 / 12} \frac{1}{2} \mu^{-1} \sin \left[\mu\left(\pi-2 \xi^{\prime}\right)\right]
$$

Expressing $\rho$ by $\eta$ and $\xi$ by means of Eqs. (1.3), one finds

$$
\psi=\rho^{-1 / 12+\mu} G(\xi, \mu) \sim \eta^{-1 / 4} \frac{1}{2} \mu^{-1} \operatorname{Im}\left\{\left[\rho e^{i\left(\pi-2 \xi^{\prime}\right)}\right]^{\mu}\right\}
$$

Similarly

$$
\psi=\rho^{-1 / 12-\mu} G(\xi, \mu) \sim \eta^{-1 / 4} \frac{1}{2} \mu^{-1} \operatorname{Im}\left\{\left[\rho e^{-i\left(\pi-2 \xi^{\prime}\right)}\right]^{-\mu}\right\}
$$

For the families of particular solutions considered, $\mu$ will assume equidistant values. Thus the terms which are essential for the convergence appear as the terms of a Taylor or Laurent development of an analytic function. Hence it follows that if $\xi<0$ the line of convergence is given by a line $\rho<\rho_{1}$, where $\rho_{1}$ is a constant. For negative values of $\mu$ the series will converge for $\rho>\rho_{1}$; for positive values of $\mu$, it will converge for $\rho>\rho_{1}$. To be more definite: if the series $\sum a_{n} \cdot \rho^{\mu_{n}}$ converges for $\rho<\rho_{1}$ and diverges for $\rho>\rho_{1}$, then the corresponding series $\sum a_{n} \cdot G\left(\xi, \mu_{n}\right) \cdot \rho^{-1 / 12+\mu_{n}}$ will converge for $\rho<\rho_{1}$ and will diverge for $\rho>\rho_{1}$; if the series $\sum b_{n} \cdot \rho^{-\mu_{n}}$ converges for $\rho>\rho_{1}$ and diverges for $\rho<\rho_{1}$ then the corresponding series $\sum b_{n} \cdot G\left(\xi, \mu_{n}\right) \cdot \rho^{-1 / 12-\mu_{n}}$ will converge for $\rho>\rho_{1}$ and will diverge for $\rho<\rho_{1}$.

b. Real values of $\mu, \xi>0$

Here the limits of the region of convergence will depend upon the presence or absence of the first term in Eq. (1.10d). If the first term is present and the powers of $\rho$ are positive,

$$
\rho^{-1 / 12+\mu} G(\xi, \mu) \sim \eta^{-1 / 4} \frac{1}{2}|\mu|^{-1}\left[\rho \frac{1+\xi^{3 / 2}}{1-\xi^{3 / 2}}\right]^{\mu} \sin \left[\pi\left(\mu+\frac{1}{4}\right)\right]
$$


By means of Eqs. (1.3) this expression is transformed into

$$
\rho^{-1 / 12+\mu} G(\xi, \mu) \sim \eta^{-1 / 4} \frac{1}{2}|\mu|^{-1}\left[\frac{3}{2} \theta+\eta^{3 / 2}\right]^{2 \mu} \sin \left[\pi\left(\mu+\frac{1}{4}\right)\right]
$$

In this equation the term important for the convergence is

$$
\left(\frac{3}{2} \theta+\eta^{\frac{3}{2}}\right)^{2 \mu}
$$

This expression is constant along a characteristic with a negative slope. If a series $\sum a_{n} \cdot \rho^{\mu_{n}}$ converges for $\rho<\rho_{1}$ and diverges for $\rho>\rho_{1}$, then for $\xi>0$ an expression $\sum a_{n} \cdot G\left(\xi, \mu_{n}\right) \cdot \rho^{-1 / 12+\mu_{n}}$ converges for $\left(\frac{3}{2} \theta+\eta^{\frac{3}{2}}\right)^{2}<\rho_{1}$ and diverges for $\left(\frac{3}{2} \theta+\eta^{\frac{3}{2}}\right)^{2}>\rho_{1}$.

These considerations do not include the line $\xi=0$; the convergence along this line could be discussed by means of Eqs. (1.14).

It is remarkable that at the supersonic limit of convergence, according to (3.1), the particular solutions are given asymptotically by the same function, i.e., by $\eta^{-\frac{1}{2}}$. const. This again shows that the application of this system of particular solutions is probably subject to rather severe restrictions. The other cases are treated similarly; the resultant regions of convergence are shown in Figures 2 and 3.

For particular solutions with imaginary values of $\mu$ and $\xi<0$ one finds

$$
\rho^{-1 / 12} \sin \{|\mu| \log \rho\} G(\xi, \mu) \sim \frac{1}{2}|\mu|^{-1} \eta^{-1} \sinh \left\{2|\mu|\left(\frac{\pi}{2}-\xi^{\prime}\right)\right\} \sin \{|\mu| \log \rho\}
$$

Here the convergence of a series is decided by the term $\sinh \left\{2|\mu|\left(\pi / 2-\xi^{\prime}\right)\right\}$. Thus it follows that in general the limit of the region of convergence is a line $\xi^{\prime}=$ const. or $\xi=$ const. If a series of particular solutions converges throughout the region $\xi<0$ for one value of $\rho$, it will converge there for any value of $\rho$.

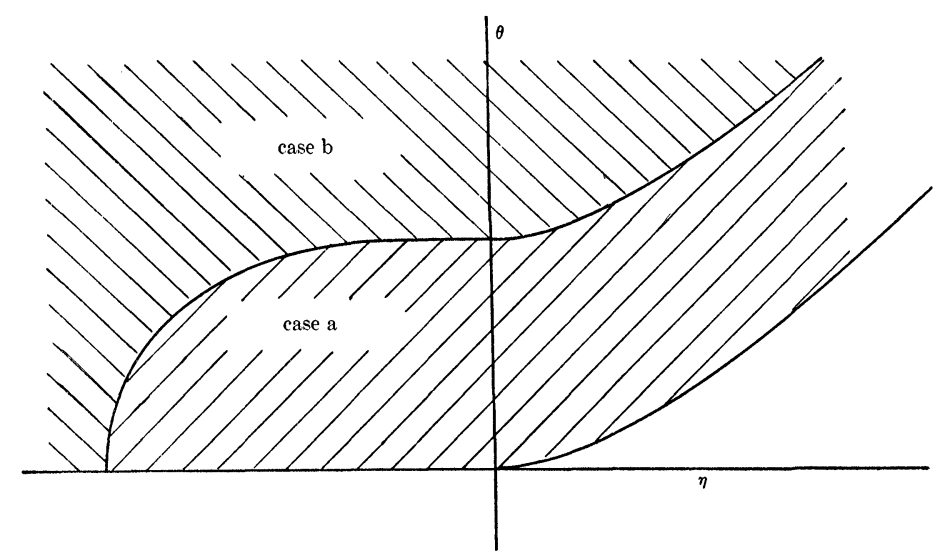

FIG. 2.

Case a. Series in positive powers of $\rho$, first term in Eq. (1.10d) absent. Case b. Series in negative powers of $\rho$, first term in Eq. (1.10d) present. 


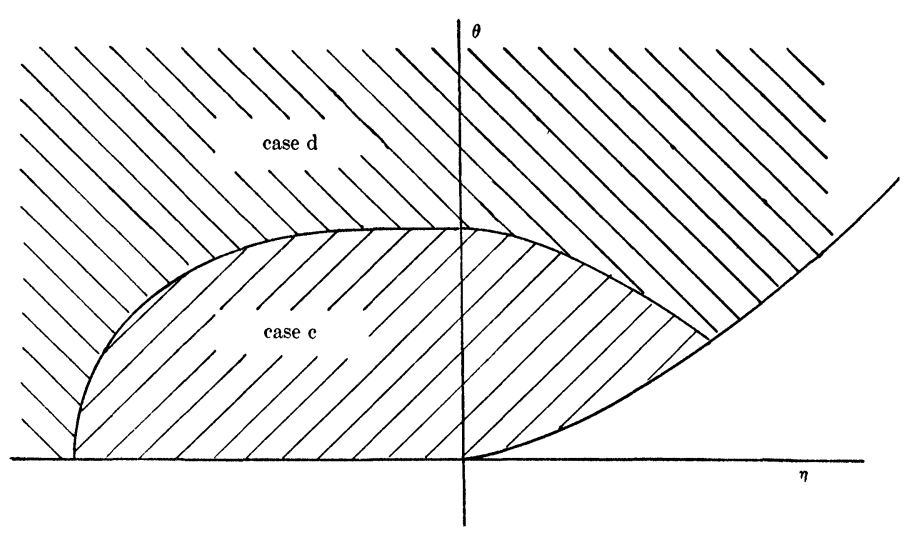

FIG. 3.

Case c. Series in positive powers of $\rho$, first term in Eq. (1.10d) present.

Case d. Series in negative powers of $\rho$, first term in Eq. (1.10d) absent.

For $\xi>0$ one finds

$G(\xi, \mu) \cdot \rho^{-1 / 12} \cos \{|\mu| \log \rho\} \sim \frac{1}{2}|\mu|^{-1} \cdot e^{\pi|\mu|} \cos \left[2|\mu|\left(\xi^{\prime}-\frac{\pi}{4}\right)\right] \sin _{\sin }^{\cos }\{|\mu| \log \rho\}$ If $\sum a_{n} \cdot e^{\pi\left|\mu_{n}\right|}$ converges absolutely then the expression

$$
\sum a_{n} G(\xi, \mu) \rho^{-1 / 12} \cos \{|\mu| \log \rho\}
$$

will converge absolutely for $0<\xi \leqq 1$. If the expression $\sum a_{n}^{2} e^{2 \pi\left|\mu_{n}\right|}$ converges, then the above expression will converge in the mean for $0<\xi<1$.

\section{Representation of an Arbitrary Function as a Superposition of Eigenfunctions}

An arbitrary function $y(\xi)$ can be represented by a Fourier series in terms of the eigenfunctions $G$. By means of the relations of orthogonality (2.4a) one finds

$$
\begin{aligned}
y(\xi)=\sum_{h=1}^{\infty} \frac{G_{h}(\xi)}{C_{h}} \int_{-\infty}^{c_{2}} y(\tau) \frac{\tau}{\left(1-\tau^{3}\right)^{7 / 6}} G_{h}(\tau) d \tau & \\
& \quad+\sum_{h=1}^{\infty} \frac{G_{-h}(\xi)}{C_{-h}} \int_{-\infty}^{c_{2}} y(\tau) \frac{\tau}{\left(1-\tau^{3}\right)^{7 / 6}} G_{-h}(\tau) d \tau
\end{aligned}
$$

where the constants $C_{h}$ are given by

$$
C_{ \pm h}=\int_{-\infty}^{c_{2}} G_{ \pm h}^{2}(\tau) \frac{\tau}{\left(1-\tau^{3}\right)^{7 / 8}} d \tau
$$

The $C_{h}$ 's are determined by the following formula (Ref. 9):

$$
\int_{-\infty}^{c_{2}} G^{2}\left(\tau, \frac{\sqrt{\lambda}}{3}\right) \frac{\tau}{\left(1-\tau^{3}\right)^{7 / 6}} d \tau=\left.\left(1-c_{2}^{3}\right)^{5 / 6}\left[G \frac{\partial^{2} G}{\partial \xi \partial \lambda}-\frac{\partial G}{\partial \xi} \frac{\partial G}{\partial \lambda}\right]\right|_{\xi=c_{3}}
$$


The expression (4.1) may now be specialized for the case where $c_{2} \rightarrow 1$. From Eq. (1.9)

$$
\mu=\frac{\sqrt{\lambda}}{3}
$$

one obtains

$$
\frac{d \mu}{d \lambda}=\frac{1}{18 \mu}
$$

Introducing for brevity

$$
B(\mu)=\frac{\Gamma\left(\frac{1}{2}\right) \Gamma(2 \mu)}{\Gamma\left(\mu+\frac{11}{12}\right) \Gamma\left(\mu+\frac{7}{12}\right)}
$$

and replacing the hypergeometric series in Eq. (1.10d) by 1, one finds for the vicinity of the point $\xi=1$

$$
\begin{aligned}
G=B(\mu) \sin \left[\pi\left(\mu+\frac{1}{4}\right)\right]\left(1-\xi^{3}\right)^{1 / 12-\mu} & \\
& +B(-\mu) \sin \left[\pi\left(-\mu+\frac{1}{4}\right)\right]\left(1-\xi^{3}\right)^{1 / 12+\mu}
\end{aligned}
$$

Since this expression remains unchanged if one interchanges $+\mu$ and $-\mu$, we can restrict ourselves to positive values of $\mu$. The boundary condition $G=0$ for $\xi=c_{2}$ yields

$$
B(\mu) \sin \left[\pi\left(\mu+\frac{1}{4}\right)\right]=-B(-\mu) \sin \left[\pi\left(-\mu+\frac{1}{4}\right)\right]\left(1-c_{2}^{3}\right)^{2 \mu}
$$

For a positive $\mu$ the limiting process $c_{2} \rightarrow 1$ yields

$$
B(\mu) \sin \left[\pi\left(\mu+\frac{1}{4}\right)\right]=0
$$

Denoting the resulting values of $\mu$ as $\mu_{h}$ one has

$$
\mu_{h}=h-\frac{1}{4} \quad h=1,2,3 \cdots
$$

The values of $\lambda$ are then found as

$$
\lambda_{h}=9 \mu_{h}^{2}=9\left(h-\frac{1}{4}\right)^{2}
$$

This definition of the eigenfunction uses only the functions $G$, while the determination of the first family of particular solutions was based on the complete expression for $\psi$. This explains why in the first family of particular solutions the results differ for positive and negative values of $\mu$, while here no difference appears. Incidentally, the particular solutions $\psi_{\mathrm{II}, h}$ as defined by Eq. (2.5b) are the same as the eigenfunctions of the first family for negative values of $\mu$. For positive values of $\mu$ there is no relation between the two families of particular solutions. 
To determine the constants $C_{h}$, we compute from Eq. (4.6)

$$
\begin{aligned}
\frac{\partial G}{\partial \xi}=B(\mu) \sin [\pi(\mu & \left.\left.+\frac{1}{4}\right)\right](-3)\left(\frac{1}{12}-\mu\right)\left(1-\xi^{3}\right)^{1 / 12-\mu} \\
& +B(-\mu) \sin \left[\pi\left(-\mu+\frac{1}{4}\right)\right](-3)\left(\frac{1}{12}+\mu\right)\left(1-\xi^{3}\right)^{1 / 12+\mu}
\end{aligned}
$$

The first terms in Eqs. (4.6) and (4.9) yield the predominant contributions in the derivatives with respect to $\lambda$, although they vanish if one inserts the eigenvalues $\mu_{h}$ from Eq. (4.7) into these equations. Using Eq. (4.4) and disregarding terms of higher order in $\left(1-\xi^{3}\right)$, one obtains

$$
\begin{aligned}
\frac{\partial G}{\partial \lambda} & =\frac{\pi}{18 \mu} B(\mu) \cos \left[\pi\left(\mu+\frac{1}{4}\right)\right]\left(1-\xi^{3}\right)^{1 / 12-\mu} \\
\frac{\partial^{2} G}{\partial \xi \partial \lambda} & =\frac{\pi}{18 \mu} B(\mu) \cos \left[\pi\left(\mu+\frac{1}{4}\right)\right](-3)\left(\frac{1}{12}-\mu\right)\left(1-\xi^{3}\right)^{-11 / 12-\mu}
\end{aligned}
$$

Taking into account that for the eigenfunctions

$$
\sin \left[\pi\left(-\mu+\frac{1}{4}\right)\right]=\cos \left[\pi\left(\mu+\frac{1}{4}\right)\right]= \pm 1
$$

one finds, by inserting Eqs. (4.6), (4.9), (4.10) and (4.11) into (4.3),

$$
C_{h}=\frac{\pi}{3} B\left(\mu_{h}\right) B\left(-\mu_{h}\right)
$$

where $\mu_{h}$ is given by (4.7) and $B(\mu)$ is defined in (4.5).

For negative values of $\lambda$, we set

$$
\mu=\frac{\sqrt{\lambda}}{3}=i \frac{\sqrt{|\lambda|}}{3}=i v
$$

then

$$
\frac{d \nu}{d \lambda}=-\frac{1}{18 \nu}
$$

Furthermore, let

$$
B(i v) \sin \left[\pi\left(i \nu+\frac{1}{4}\right)\right]=A(\nu) e^{i \sigma(v)}
$$

where $A(\nu)$ and $\sigma(\nu)$ are real. Since $B(\mu)$ and $\sin \left[\pi\left(\mu+\frac{1}{4}\right)\right]$ are analytic functions which are real for real values of $\mu$, because of ScHwanz's principle of symmetry, the expression $B(-i \nu) \sin \left[\pi\left(-i \nu+\frac{1}{4}\right)\right]$ is the conjugate complex of

$$
B(i \nu) \sin \left[\pi\left(i \nu+\frac{1}{4}\right)\right] .
$$

Thus one obtains from (4.6)

$$
G=2 A(\nu)\left(1-\xi^{3}\right)^{1 / 12} \cos \left[\sigma(\nu)-\nu \log \left(1-\xi^{3}\right)\right]
$$

The boundary condition $G=0$ for $\xi=c_{2}$ yields

$$
\cos \left[\sigma\left(\nu_{h}\right)-\nu_{h} \log \left(1-c_{2}^{3}\right)\right]=0
$$


where $\nu_{h}$ denotes the value of $\nu$ for the $h^{\text {th }}$ negative eigenvalue. Using this equation and carrying out the limiting process $c_{2} \rightarrow 1$ in some terms, one finds

$$
\begin{aligned}
& \frac{\partial G}{\partial \lambda}=\frac{1}{9} \frac{A}{\nu_{h}}\left(1-c_{2}^{3}\right)^{1 / 12} \sin \left[\sigma\left(\nu_{h}\right)-\nu_{h} \log \left(1-c_{2}^{3}\right)\right]\left(\frac{d \sigma}{d \nu}-\log \left(1-c_{2}^{3}\right)\right) \\
& \frac{\partial G}{\partial \xi}=-6 A\left(1-c_{2}^{3}\right)^{-11 / 12} \nu_{h} \sin \left[\sigma\left(\nu_{h}\right)-\nu_{h} \log \left(1-c_{2}^{3}\right)\right]
\end{aligned}
$$

Because of Eq. (4.17) the absolute value of the sine function in the last two equations is 1 . For $\xi=c_{2}$ one has $G=0$. Thus one finds from Eq. (4.3)

$$
C_{-h}=\frac{2}{3} A^{2}\left(\nu_{h}\right)\left[\frac{d \sigma}{d \nu}-\log \left(1-c_{2}^{3}\right)\right]
$$

$C_{h}$ tends to positive infinity as $\left(1-c_{2}^{3}\right)$ tends to zero.

The values of $\nu_{h}$ are determined from Eq. (4.17):

$$
\sigma\left(\nu_{h}\right)-\nu_{h} \log \left(1-c_{2}^{3}\right)=\pi\left(\frac{1}{2}+h\right)
$$

For small values of $\left(1-c_{2}^{3}\right)$ the quantity $\nu_{h}$ will change by only a small amount if $h$ changes by unity. Denoting by $\Delta \nu_{h}$ the change of $\nu_{h}$ between two consecutive values of $h$, one obtains from the last equation

$$
\Delta \nu_{h}\left[\frac{d \sigma}{d \nu_{h}}-\log \left(1-c_{2}^{3}\right)\right] \rightarrow \pi \quad \text { as } \quad\left(1-c_{2}^{3}\right) \rightarrow 0
$$

With this formula and with Eq. (4.18) the second expression occurring in Eq. (4.1) can be changed from a summation over $h$ to a summation over the $\Delta \nu_{h}$

$$
\begin{aligned}
\sum_{h=1}^{\infty} \frac{G_{-h}}{C_{-h}} \int_{-\infty}^{c_{2}} y(\tau) \frac{\tau}{\left(1-\tau^{3}\right)^{7 / 6}} G_{-h}(\tau) d \tau & \\
& =\sum_{\Delta \nu_{h}} \frac{\Delta \nu_{h} G\left(\xi, i \nu_{h}\right)}{\frac{2}{3} \pi A^{2}\left(\nu_{h}\right)} \int_{-\infty}^{c_{2}} y(\tau) \frac{\tau}{\left(1-\tau^{3}\right)^{7 / 6}} G\left(\tau, i \nu_{h}\right) d \tau
\end{aligned}
$$

Hence one finds

$$
\begin{aligned}
\lim _{c_{2} \rightarrow 1} \sum_{h=1}^{\infty} \frac{G_{-h}}{C_{-h}} \int_{-\infty}^{c_{2}} y(\tau) & \frac{\tau}{\left(1-\tau^{3}\right)^{7 / 6}} G_{-h}(\tau) d \tau \\
& =\frac{3}{2 \pi} \int_{0}^{\infty} d \nu \frac{G(\xi, i \nu)}{A^{2}(\nu)} \int_{-\infty}^{1} y(\tau) \frac{\tau}{\left(1-\tau^{3}\right)^{7 / 6}} G(\tau, i \nu) d \tau
\end{aligned}
$$


These results are inserted into Eq. (4.1). In particular we express $C_{h}$ by Eq. (4.12) replace the second expression in (4.1) by (4.19) and insert $A$ from Eq. (4.15). Furthermore, we rewrite the integration over $\nu$ which occurs in Eq. (4.19) as an integration over $\mu$; then, according to Eq. (4.13), the integration will take place along the imaginary axis of the complex $\mu$-plane:

$$
\begin{array}{r}
y(\xi)=\frac{3}{\pi}\left\{\sum_{h=1}^{\infty} \frac{G\left(\xi, \mu_{h}\right)}{B\left(\mu_{h}\right) B\left(-\mu_{h}\right)} \int_{-\infty}^{1} y(\tau) \frac{\tau}{\left(1-\tau^{3}\right)^{7 / 6}} G(\tau, \mu) d \tau\right. \\
-\frac{i}{2} \int_{\mu=0}^{i \infty}\left\{\frac{G(\xi, \mu)}{B(\mu) B(-\mu) \sin \left[\pi\left(\mu+\frac{1}{4}\right)\right] \sin \left[\pi\left(-\mu+\frac{1}{4}\right)\right]}\right. \\
\left.\cdot \int_{-\infty}^{1} y(\tau) \frac{\tau}{\left(1-\tau^{3}\right)^{7 / 6}} G(\tau, \mu) d \tau\right\} d \mu
\end{array}
$$

here

$$
\mu_{h}=h-\frac{1}{4}
$$

and

$$
B(\mu)=\frac{\Gamma\left(\frac{1}{2}\right) \Gamma(2 \mu)}{\Gamma\left(\mu+\frac{1}{12}\right) \Gamma\left(\mu+\frac{7}{12}\right)}
$$

\section{On the Representation of an Arbitrary Solution of Tricomi's Equation}

It is the aim of the present paper to show that an arbitrary solution of TRIcomr's equation can be expressed, in a suitable region and under conditions which will become obvious from the derivations below, as a superposition of particular solutions of the first family.

Obviously the second family of particular solutions is more general and, indeed, our investigations will use it as a starting point.

In general the functions $\psi$ in which we are interested arise by the solution of a boundary value problem, e.g. a boundary value problem of TRICOMI's type. However, we are not concerned at present with the construction of the solution of such a boundary value problem: we assume that the solution is known. Actually it will suffice to know $\psi$ and $\psi_{\rho}$ along a certain curve $C$, say the boundary of the original boundary value problem.

To fix the ideas let the curve $C$ be defined by $\rho=\tilde{\rho}(\xi)$ for $-\infty<\xi \leqq 1$. For simplicity $\tilde{\rho}(\xi)$ may be considered as a one-valued function. A point with coordinates $\rho$, $\xi$ such that $\rho<\tilde{\rho}(\xi)$ will be denoted as a point of the inner region. If $\rho>\tilde{\rho}(\xi)$, we speak of the outer region.

One notices that $\psi$ and $\psi_{\rho}$ cannot be prescribed independently along the curve $C$, so if these quantities are given arbitrarily, no function can be found which satisfies Eq. (1.6) and assumes these values along $C$. On the other hand it would amount to the solution of the boundary value problem if one were to establish the relation existing between the values of $\psi$ and $\psi_{\rho}$ along the curve $C$.

To overcome this difficulty we consider a different problem in which it is 
possible to prescribe quantities which are equivalent to $\psi$ and $\psi_{\rho}$ independently; this problem will have a unique solution for an arbitrary choice of these new quantities. In the case where the relations between $\psi$ and $\psi_{\rho}$ mentioned above are satisfied, this problem will have as a solution the function $\psi$ of the original boundary value problem. For this purpose we define a function $\bar{\psi}$ which coincides in the inner region with the function $\psi$ which we wish to represent, and which has $\bar{\psi} \equiv 0$ in the outer region. At the curve $C$ we have a jump in $\bar{\psi}$ and in $\bar{\psi}_{\rho}$. Now we seek the solution of the inhomogeneous boundary value problem where $\bar{\psi}$ vanishes at infinity and fulfills at the origin the conditions of TRICOMI's uniqueness and existence proof, and which has at the curve $C$ the jumps in $\bar{\psi}$ and $\bar{\psi}_{\rho}$ found from the above construction of $\bar{\psi}$. This boundary value problem has a unique solution even if one chooses the functions which give arbitrary jumps in $\bar{\psi}$ and $\bar{\psi}_{\rho}$. If the functions representing the jumps in $\bar{\psi}$ and $\bar{\psi}_{\rho}$ fit together, e.g., if they are taken from a function which satisfies Tricomi's differential equation, then one obtains in the outer region $\bar{\psi} \equiv 0$. If they do not fit together, then the function $\bar{\psi}$ thus obtained will be different from zero in the outer region but a solution will exist in any case.

In the following sections it will be shown that under certain conditions the solution of the inhomogeneous boundary value problem just described can be expressed, in the vicinity of the origin, by a superposition of the functions of the first system. If this is the case, then any function $\bar{\psi}$ which fulfills these conditions can be represented in this manner. For simplicity the function $\bar{\psi}$ will be denoted in the following by $\psi$.

\section{The Inhomogeneous Boundary Value Problem}

Only such solutions $\psi$ as are antisymmetric with respect to the axis $\xi=-\infty$ will be considered. The region considered extends then throughout the upper part of the $\eta, \theta$-plane with the exception of the region which lies beneath the characteristic $0 A$ (Fig. 1). In the $\rho, \xi$-plane where the actual computations are carried out, the region considered extends between $\rho=0$ and $\rho=\infty$ and $\xi=-\infty$ and $\xi=1$.

With regard to the curve $C$ defined in the previous paragraph we mention only that in general $\tilde{\rho}(\xi)$ is bounded and that if in the $\eta, \theta$-plane the curve $C$ ends at a finite distance from the origin along the characteristic $0 A$ of Fig. 1, $\rho$ tends to zero as $\xi$ tends to 1 .

We have the following problem: We must determine a function $\psi(\rho, \xi)$ which satisfies everywhere in the region indicated the differential equation (1.6), which vanishes along $\xi=-\infty$ (because of the condition of antisymmetry), and which vanishes at infinity of the $\eta, \theta$-plane, i.e., it vanishes for $\rho \rightarrow \infty$ and for $\xi \rightarrow 1$ if $\rho \neq 0$. Furthermore it fulfills the conditions of TrICOMI's uniqueness and existence theorem at the origin and has at the curve $C$ a jump of $\psi$ and $\psi_{\rho}$ given by two functions $f_{1}(\xi)$ and $f_{2}(\xi)$. Using superscripts $i$ and o in order to differentiate between the inner and the outer region, one thus has the "jump conditions" 


$$
\begin{aligned}
& \psi^{\mathrm{i}}(\tilde{\rho}(\xi), \xi)-\psi^{\circ}(\tilde{\rho}(\xi), \xi)=f_{1}(\xi) \\
& \psi_{\rho}^{\mathrm{i}}(\tilde{\rho}(\xi), \xi)-\psi^{\circ}(\tilde{\rho}(\xi), \xi)=f_{2}(\xi)
\end{aligned}
$$

Before we start the treatment of this problem we introduce a few notations. The inverse of the function $\tilde{\rho}(\xi)$ will be denoted by $\xi=\tilde{\xi}(\rho)$. The derivative of this function with respect to its argument will be denoted by $\xi^{*}$. Derivatives with respect to $\xi$ which occur in $\tilde{\rho}$ and in $G$ will be denoted by a prime. The function $\tilde{\xi}(\rho)$ need not be one-valued. However, it will be assumed that along the curve $C \rho$ assumes the value 0 only once, viz. for $\xi=1$. Most of our investigations will be concerned with the vicinity of $\xi=1$ and there $\tilde{\xi}$ will be a onevalued function. Whenever it is necessary, the different values which $\tilde{\xi}(\rho)$ can assume for a given value of $\rho$ will be denoted by superscript $k(k=1,2$ etc.).

Naturally one has

$$
\frac{d \tilde{\xi}}{d \rho} \frac{d \tilde{\rho}}{d \xi}=\tilde{\xi} \cdot \tilde{\rho}^{\prime}=1
$$

From Eqs. (5.1) and (5.2) one obtains an expression for the jump of the derivatives with respect to $\xi$ at the curve $C$. Differentiation of Eq. (5.1) with respect to $\xi$ yields

$$
\psi_{\xi}^{\mathrm{i}}(\tilde{\rho}(\xi), \xi)-\psi_{\xi}^{0}(\tilde{\rho}(\xi), \xi)+\left[\psi_{\rho}^{\mathrm{i}}(\tilde{\rho}(\xi), \xi)-\psi_{\rho}^{\circ}(\tilde{\rho}(\xi), \xi)\right] \tilde{\rho}^{\prime}=f_{1}^{\prime} ;
$$

hence with Eq. (5.2)

$$
\psi_{\xi}^{\mathrm{i}}(\tilde{\rho}(\xi), \xi)-\psi_{\xi}^{\circ}(\tilde{\rho}(\xi), \xi)=f_{1}^{\prime}-f_{2} \tilde{\rho}^{\prime}
$$

In order to construct the desired function $\psi$ we first choose an auxiliary function $\chi$, which need not satisfy the differential equation (1.6) but otherwise fulfills all conditions imposed on $\psi$; in particular the jump relations (5.1), (5.2) and (5.4) are satisfied if one replaces $\psi$ by $\chi$.

For later use the following expression $c(\rho, \mu)$ may be introduced; it would occur in a Fourier representation of the function $\chi$ by means of Eq. (4.20)

$$
c(\rho, \mu)=\int_{-\infty}^{1} \frac{\tau}{\left(1-\tau^{3}\right)^{7 / 6}} \chi(\rho, \tau) G(\tau, \mu) d \tau
$$

The integration is to be carried out along the line $\rho=$ const. corresponding to the first argument of $c(\rho, \mu)$. Certain integrals which will be used later can be expressed in terms of $c$. In the following transformations one must keep in mind that $\chi$ and its derivatives are not continuous at the points of intersection of the line $\rho=$ const. along which the integration is carried out and the curve $C$. To emphasize this, the following integral is first written as the integral over several intervals in which $\chi$ and $\chi_{\xi}$ are continuous. Afterwards an integration by parts is carried out 


$$
\begin{aligned}
& \int_{-\infty}^{1} G(\tau, \mu) \frac{\partial}{\partial \tau}\left[\left(1-\tau^{3}\right)^{5 / 6} \chi_{\tau}(\rho, \tau)\right] d \tau \\
& =\int_{-\infty}^{\substack{(\hat{)}) \\
\xi(\rho)}}+\int_{\substack{(\underline{1}) \\
\xi(\rho)}}^{\substack{(\xi) \\
\xi(\rho)}}+\int_{\substack{(n) \\
\xi(\rho)}}^{1} G(\tau, \mu) \frac{\partial}{\partial \tau}\left[\left(1-\tau^{3}\right)^{5 / 6} \chi_{\tau}(\rho, \tau)\right] d \tau \\
& =\left.G(\tau, \mu)\left(1-\tau^{3}\right)^{5 / 6} \chi_{\tau}(\tau, \rho)\right|_{-\infty} ^{\substack{(1) \\
\xi(\rho)}}+\left.\right|_{\substack{(1) \\
\xi(\rho)}} ^{\substack{(\rho) \\
\xi(\rho)}} \cdots+\left.\right|_{\substack{(n) \\
\xi(\rho)}} ^{1} \\
& -\int_{-\infty}^{1} G^{\prime}(\tau, \mu)\left(1-\tau^{3}\right)^{5 / 6} \chi_{\tau}(\rho, \tau) d \tau
\end{aligned}
$$

According to the assumptions made, $\psi$ and $\chi$ are of such a nature that for $\xi=-\infty$ and $\xi=1$ the terms outside of the integral vanish. The combination of the upper limit of one term outside of the integral with the lower limit of the next term gives the jump in $\chi_{\xi}$ or $\psi_{\xi}$ which one experiences along a line $\rho=$ const. at a point of an intersection with the curve $C$. Thus these terms can be expressed by means of Eq. (5.4). Regarding the sign of the terms which thus arise, we can state that traveling along a line $\rho=$ const. in the direction of increasing $\xi$, one moves according to Fig. 4 from the outer to the inner region if $\tilde{\rho}^{\prime}$ is positive. The opposite is the case if $\tilde{\rho}^{\prime}$ is negative. Carrying out simultaneously a further integration by parts, one finds

$$
\begin{aligned}
& \int_{-\infty}^{1} G(\tau, \mu) \frac{\partial}{\partial \tau}\left[\left(1-\tau^{3}\right)^{5 / 6} \chi_{\tau}(\rho, \tau)\right] d \tau
\end{aligned}
$$

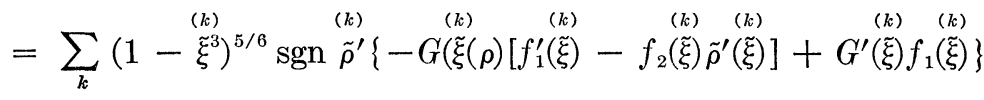

$$
\begin{aligned}
& +\int_{-\infty}^{1} \frac{d}{d \tau}\left[G^{\prime}(\tau, \mu)\left(1-\tau^{3}\right)^{5 / 6}\right] \chi(\rho, \tau) d \tau
\end{aligned}
$$

(b)

Here $\tilde{\xi}$ is a function of $\rho$.

In the last integral, Eq. (1.7) together with (1.9) may be introduced. The integral which then arises is the expression (5.5). Thus one finally obtains

$$
\begin{aligned}
& \int_{-\infty}^{1} G(\tau, \mu) \frac{\partial}{\partial \tau}\left[\left(1-\tau^{3}\right)^{5 / 6} \chi_{\tau}(\rho, \tau)\right] d \tau \\
& \left.=\sum_{k=1}^{n}\left(1-\stackrel{(k)}{\xi^{3}}\right)^{5 / 6} \operatorname{sgn} \stackrel{(k)}{\tilde{\rho} \cdot} \cdot\left\{-G^{(\xi)}, \mu\right)\left[\stackrel{(k)}{f_{1}^{\prime}(\tilde{\xi})}-f_{2}^{\prime(k)}(\tilde{\xi}) \tilde{\rho}^{\prime}(\tilde{\xi})\right]+G^{\prime}(\tilde{\xi}, \mu) f_{1}^{(k)}(\tilde{\xi})\right\} \\
& +\left(9 \mu^{2}-\frac{1}{16}\right) c(\rho, \mu)
\end{aligned}
$$


In this formula the function $\chi$ enters only the last expression. All other terms are expressed by data of the original boundary value problem.

Let us furthermore carry out the differentiations occurring in the following expression

$$
\frac{d}{d \rho}\left[\rho^{7 / 6} \frac{d}{d \rho} c(\rho, \mu)\right]=\frac{d}{d \rho}\left[\rho^{7 / 6} \frac{d}{d \rho} \int_{-\infty}^{1} \frac{\tau}{\left(1-\tau^{3}\right)^{7 / 6}} G(\tau, \mu) \chi(\rho, \tau) d \tau\right]
$$

Since the integrand is discontinuous at the points $\tau=\tilde{\xi}(\rho)$ and since the location of these discontinuities depends upon $\rho$, one must treat this integral as the sum of integrals over intervals in which $\chi$ is continuous. The differentiation with respect to $\rho$ is then carried out with respect to the integrand as well as with respect to the limits of integration. Some simplifications are obtained by considerations similar to those made in the derivation of Eq. (5.6).

$$
\begin{aligned}
& \frac{d}{d \rho}\left[\rho^{7 / 6} \frac{d c}{d \rho}\right]=\sum_{k}-\frac{d}{d \rho}\left\{\rho^{7 / 6} \frac{\stackrel{(k)}{\xi}}{\left(1-\xi^{(k)}\right)^{7 / 6}} G(\tilde{\xi}, \mu) f_{1}(\tilde{\xi}) \operatorname{sgn} \tilde{\xi}^{(k)} \stackrel{(k)}{\tilde{\xi}^{*}}\right\} \\
& +\frac{d}{d \rho}\left[\rho^{7 / 6} \int_{-\infty}^{1} \frac{\tau}{\left(1-\tau^{3}\right)^{7 / 6}} G(\tau, \mu) \chi_{\rho}(\rho, \tau) d \tau\right]
\end{aligned}
$$

and hence

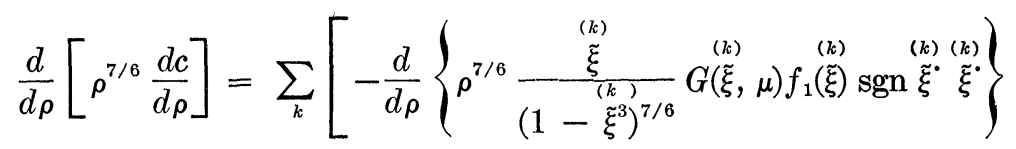

$$
\begin{aligned}
& \left.-\rho^{7 / 6} \frac{\stackrel{(k)}{\tilde{\xi}}}{\left(1-\stackrel{(k)}{\tilde{\xi}}^{3}\right)^{7 / 6}} G(\stackrel{(k)}{(\tilde{\xi}}, \mu) f_{2}(\stackrel{(k)}{(\tilde{\xi})}) \operatorname{sgn}{\stackrel{(k)}{\tilde{\xi}^{\cdot}}}^{\stackrel{(k)}{\xi^{*}}}\right] \\
& +\int_{-\infty}^{1} \frac{\tau}{\left(1-\tau^{3}\right)^{7 / 6}} G(\tau, \mu) \frac{d}{d \rho}\left(\rho^{7 / 6} \chi_{\rho}(\rho, \tau) d \tau\right.
\end{aligned}
$$

Now the following hypothesis, patterned after equation (4.20), is made for the solution of the boundary value problem formulated above;

$$
\begin{aligned}
\psi(\rho, \xi)=\chi(\rho, \xi)+ & \frac{3}{\pi}\left\{\sum_{h=1}^{\infty} \frac{G\left(\xi, \mu_{h}\right)}{B\left(\mu_{h}\right) B\left(-\mu_{h}\right)} a\left(\rho, \mu_{h}\right)\right. \\
& \left.-\frac{i}{2} \int_{\mu=0}^{i \infty} \frac{a(\rho, \mu) G(\xi, \mu) d \mu}{B(\mu) B(-\mu) \sin \left[\pi\left(\mu+\frac{1}{4}\right)\right] \sin \left[\pi\left(-\mu+\frac{1}{4}\right)\right]}\right\}
\end{aligned}
$$

The functions $a(\rho, \mu)$ are unknown up to now. They will be determined subsequently by means of the differential equation for $\psi$, Eq. (1.6). Since all discontinuities of $\psi$ are contained in $\chi$, the function represented by the terms in the braces 
will be such that the differentiations required in Eq. (1.6) can be carried out under the summation and under the integral sign. Using Eq. (1.7) as needed, one obtains

$$
\begin{aligned}
\frac{d}{d \xi}\left[\left(1-\xi^{3}\right)^{5 / 6} \chi_{\xi}\right]- & \frac{9 \xi}{\left(1-\xi^{3}\right)^{7 / 6}} \rho^{5 / 6} \frac{\partial}{\partial \rho}\left(\rho^{7 / 6} \chi_{\rho}\right) \\
=-\frac{3}{\pi} & \frac{\xi}{\left(1-\xi^{3}\right)^{7 / 6}}\left\{\sum_{h=1}^{\infty} \frac{\left(9 \mu_{h}^{2}-\frac{1}{16}\right)}{B\left(\mu_{h}\right) B\left(-\mu_{h}\right)} G\left(\xi, \mu_{h}\right) a\left(\rho, \mu_{h}\right)\right. \\
& -\frac{i}{2} \int_{0}^{i \infty} \frac{\left(9 \mu^{2}-\frac{1}{16}\right) G(\xi, \mu) a(\rho, \mu) d \mu}{B(\mu) B(-\mu) \sin \left[\pi\left(\mu+\frac{1}{4}\right)\right] \sin \left[\pi\left(-\mu+\frac{1}{4}\right)\right]} \\
& -\sum_{h=0}^{\infty} \frac{G\left(\xi, \mu_{h}\right)}{B\left(\mu_{h}\right) B\left(-\mu_{h}\right)} 9 \rho^{5 / 6} \frac{d}{d \rho}\left(\rho^{7 / 6} \frac{d a(\rho, \mu)}{d \rho}\right) \\
& \left.+\frac{i}{2} \int_{\mu=0}^{\infty} \frac{G(\xi, \mu) 9 \rho^{5 / 6} \frac{d}{d \rho}\left(\rho^{7 / 6} \frac{d a(\rho, \mu)}{d \rho}\right) d \mu}{B(\mu) B(-\mu) \sin \left[\pi\left(\mu+\frac{1}{4}\right)\right] \sin \left[\pi\left(-\mu+\frac{1}{4}\right)\right]}\right\}
\end{aligned}
$$

This equation holds everywhere in the region of the $\rho, \xi$-plane considered. Now we express also the terms on the left hand side by means of Eq. (4.20). The integrals over $\tau$ which are required for this purpose are expressed in Eqs. (5.6) and (5.7). Equating the coefficients of the terms $G(\xi, \mu)$ which will then appear on the left and right side of the equation, one finds the following ordinary differential equation for the functions $a(\rho, \mu)$

$$
\begin{aligned}
& 9 \rho^{5 / 6} \frac{d}{d \rho}\left[\rho^{7 / 6} \frac{d a(\rho, \mu)}{d \rho}\right]-\left(9 \mu^{2}-\frac{1}{16}\right) a(\rho, \mu) \\
& =-9 \rho^{5 / 6} \frac{d}{d \rho}\left[\rho^{7 / 6} \frac{d c(\rho, \mu)}{d \rho}\right]+\left(9 \mu^{2}-\frac{1}{16}\right) c(\rho, \mu)
\end{aligned}
$$

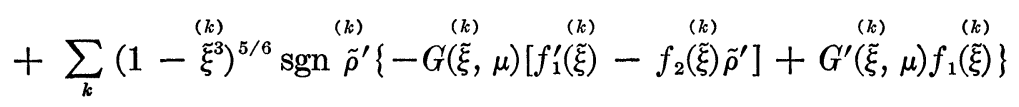

$$
\begin{aligned}
& +9 \rho^{5 / 6} \sum_{k}-\frac{d}{d \rho}\left[\rho^{7 / 6} \frac{\stackrel{(k)}{\tilde{\xi}}}{\left(1-\stackrel{(k)}{\tilde{\xi}}^{3}\right)^{7 / 6}} G(\xi, \mu) f_{1}(\stackrel{(k)}{\xi}) \operatorname{sgn} \stackrel{(k)}{\xi}^{\cdot} \stackrel{(k)}{\tilde{\xi}^{*}}\right] \\
& -9 \rho^{5 / 6} \sum_{k} \rho^{7 / 6} \frac{\stackrel{(k)}{\xi}}{\left(1-{\left.\stackrel{(k)}{\xi})^{3}\right)^{7 / 6}}_{(k)}^{(k)}\right.} G(\tilde{\xi}, \mu) f_{2}(\stackrel{(k)}{\xi}) \operatorname{sgn} \stackrel{(k)}{\xi^{*}} \stackrel{(k)}{\xi}^{*}
\end{aligned}
$$

The solutions $a(\rho, \mu)$ of this differential equation may be written in the form

$$
a(\rho, \mu)=a_{1}(\rho, \mu)+a_{2}(\rho, \mu)+a_{3}(\rho, \mu)
$$

where $a_{1}(\rho, \mu)$ is the solution of the above differential equation if all terms on the right hand side are omitted except those originating from $f_{1}$, and where 
$a_{2}$ and $a_{3}$ are the corresponding expressions due to $f_{2}$ and to $c(\rho, \mu)$. The solution for $a_{3}$ is immediately obvious:

$$
a_{3}(\rho, \mu)=-c(\rho, \mu)
$$

If in Eq. (5.8) the function $\chi$ is developed by means of Eq. (4.20), then according to the last equation this development cancels the terms $a_{3}$. Thus the resulting expression $\psi$ will contain only the contributions due to $a_{1}$ and $a_{2}$. The general solution of the following differential equation, whose homogeneous part agrees with that of Eq. (5.9),

$$
\rho^{5 / 6} \frac{d}{d \rho}\left(\rho^{7 / 6} \frac{d a}{d \rho}\right)-\left(\mu^{2}-\frac{1}{144}\right) a(\rho)=R(\rho)
$$

is given by

$a(\rho)=\frac{1}{2 \mu}\left\{\rho^{-1 / 12+\mu} \int_{c_{1}}^{\rho} u^{-11 / 12-\mu} R(u) d u-\rho^{-1 / 12-\mu} \int_{c_{2}}^{\rho} u^{-11 / 12+\mu} R(u) d u\right\}$

It can be derived by the method of variation of the parameters or verified directly. A change of the constants of integration $c_{1}$ and $c_{2}$ is equivalent to the addition of a solution of the homogeneous equation. To fulfill the conditions at infinity and at the origin of the plane the following choice of the constant $c_{1}$ and $c_{2}$ will be made:

$$
\begin{array}{ll}
\text { for real positive values of } \mu & c_{1}=\infty, c_{2}=0 \\
\text { for imaginary values of } \mu & c_{1}=\infty, c_{2}=\infty
\end{array}
$$

It is immediately obvious that with this choice of the constants of integration the function $\psi$ will vanish at infinity. That its behavior at the origin fulfills TRICOMI's conditions can be seen at the end of the present investigations when the solution appears as a superposition of particular solutions of the first family.

According to Eq. (5.12) one finds for $a_{2}$

$$
\begin{aligned}
& a_{2}=\frac{1}{2 \mu}\left\{\rho^{-1 / 12+\mu} \int_{\infty}^{\rho} u^{13 / 12-\mu} \sum_{k} \frac{\stackrel{(k)}{\xi}(u)}{\left(1-\stackrel{(k)}{\tilde{\xi}(}(u)^{3}\right)^{7 / 6}}\right.
\end{aligned}
$$

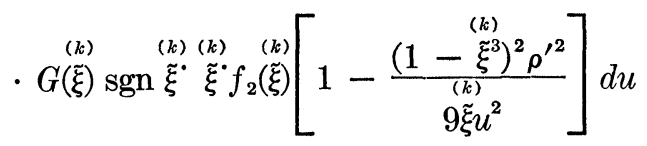

$$
\begin{aligned}
& +\rho^{-1 / 12-\mu} \int_{0}^{\rho} u^{13 / 12+\mu} \sum_{k} \frac{\stackrel{(k)}{\tilde{\xi}(u)}}{\left(1-\stackrel{(k)}{\left.\tilde{\xi}(u)^{3}\right)^{7 / 6}}\right.}
\end{aligned}
$$

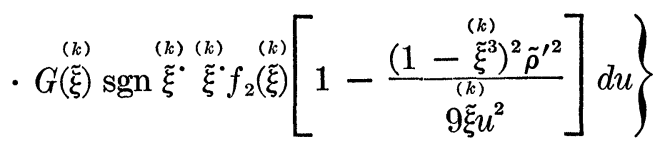


As is obvious from the limits of the integrals, the variable $u$ expresses an integration over $\rho$. The integrands require an evaluation along the curve $C$. It is more practical to choose as independent variable a quantity related to $\xi$

We set

$$
v=\tilde{\xi}(u) .
$$

Since $\tilde{\rho}$ is the inverse of the function $\tilde{\xi}$, we have $u=\tilde{\rho}(v)$. Hence $d u=\tilde{\rho}^{\prime} d v$. Furthermore we have the relation (5.3) viz. $\tilde{\rho}^{\prime} \cdot \tilde{\xi}^{\cdot}=1$. The transformation of the limits of integration requires some attention. Consider the first integral which extends from $u=\infty$ to $\rho$. Interchanging the limits of integration and changing the sign of the integral, one obtains an integral for which the integration proceeds in the positive $u$-direction. The integrand contains only values which are defined along the curve $C$. The integration is to be performed for portions of $C$ for which $u=\tilde{\rho}$ is larger than $\rho$. For an integration which proceeds in the positive $u$ direction the lower limits of the integral are the points $A B C D$ of Figure 4; the upper limits are the maxima of the curve. Now we interchange the direction of integration for all portions of $C$ for which $\tilde{\rho}^{\prime}$ is negative. The necessary change of sign will cancel the term $\operatorname{sgn} \stackrel{(k)}{\xi}^{\cdot}$ in the integral, and the integral will proceed everywhere in the positive $v$-direction, but naturally only for those portions of the curve for which $\tilde{\rho}>\rho$. The treatment of the second integral in Eq. (5.13) is quite similar. Thus one obtains for real positive values of $\mu$

$$
\begin{aligned}
& a_{2}=\frac{1}{2 \mu}\left\{\rho^{-1 / 12+\mu} \int_{\substack{\tilde{\rho}>\rho \\
\rho}}^{1} \tilde{\rho}(v)^{13 / 12-\mu} \frac{v}{\left(1-v^{3}\right)^{7 / 6}}\right. \\
& \cdot G(v, \mu) f_{2}(v)\left[1-\frac{\left(1-v^{3}\right)^{2}}{9 v} \frac{\tilde{\rho}^{\prime}(v)^{2}}{\tilde{\rho}(v)^{2}}\right] d v \\
&+\rho^{-1 / 12-\mu} \int_{-\infty}^{1} \tilde{\rho}(v)^{13 / 12+\mu} \frac{v}{\left(1-v^{3}\right)^{7 / 6}} \\
&\left.\cdot G(v, \mu) f_{2}(v)\left[1-\frac{\left(1-v^{3}\right)^{2}}{9 v} \cdot \frac{\tilde{\rho}^{\prime}(v)^{2}}{\tilde{\rho}(v)^{2}}\right] d v\right\}
\end{aligned}
$$

For imaginary values of $\mu$ the procedure is the same, except that the limits of the second integral are different; one finds

$$
\begin{aligned}
a_{2}=\frac{1}{2 \mu} & \left\{\rho^{-1 / 12+\mu} \int_{\substack{\tilde{\rho}>\rho \\
\tilde{\rho}>\rho}}^{1} \tilde{\rho}(v)^{13 / 12-\mu} \frac{v}{\left(1-v^{3}\right)^{7 / 6}} G(v, u) f_{2}(v)\left[1-\frac{\left(1-v^{3}\right)^{2}}{9 v} \frac{\tilde{\rho}^{\prime}(v)^{2}}{\tilde{\rho}(v)^{2}}\right] d v\right. \\
& \left.-\rho^{-1 / 12-\mu} \int_{\substack{-\infty \\
\tilde{\rho}>\rho}}^{1} \tilde{\rho}(v)^{13 / 12+\mu} \frac{v}{\left(1-v^{3}\right)^{7 / 6}} G(v, \mu) f_{2}(v)\left[1-\frac{\left(1-v^{3}\right)^{2}}{9 v} \frac{\tilde{\rho}^{\prime}(v)^{2}}{\tilde{\rho}(v)^{2}}\right] d v\right\}
\end{aligned}
$$




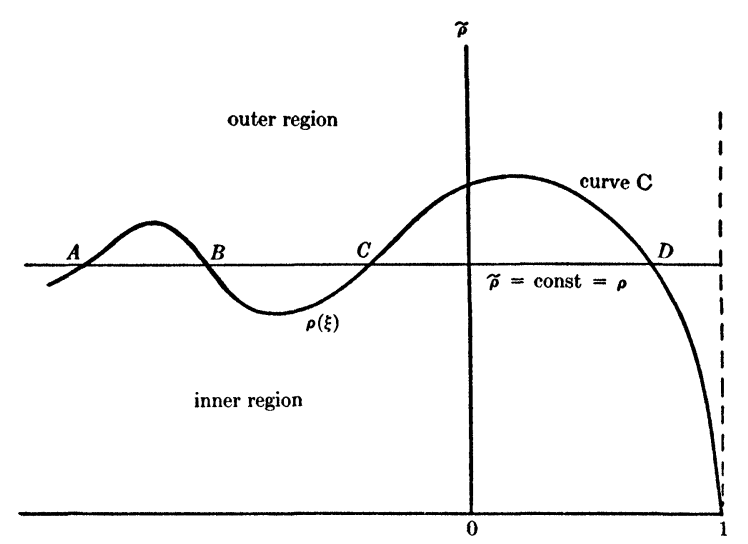

Frg. 4.

Because of Schwarz's principle of symmetry the two terms which occur in the brackets are conjugate complex. Thus the last expression can also be written as

$$
a_{2}=\operatorname{Re}\left\{\frac{1}{\mu} \rho^{-1 / 12+\mu} \int_{\substack{\tilde{\rho}>\rho \\ \rho}}^{1} \tilde{\rho}(v)^{13 / 12-\mu} \frac{v}{\left(1-v^{3}\right)^{7 / 6}} G(v, \mu) f_{2}(v)\left[1-\frac{\left(1-v^{3}\right)^{2}}{9 v} \frac{\tilde{\rho}^{\prime}(v)^{2}}{\tilde{\rho}(v)^{2}}\right] d v\right\}
$$

If the curve $C$ extends along a characteristic, the bracket in the last equation will vanish, as can be seen if one determines the slopes of the characteristics from Eq. (1.5). Thus the solution is independent of the choice of the function $f_{2}$ in such intervals. Actually it is clear that something peculiar must happen in such a case, for along a characteristic the functions $f_{1}$ and $f_{2}$ cannot be prescribed independently. According to the last result $f_{2}$ does not influence the function $\psi$ at all. What happens in this case can be visualized if we consider an example for which $f_{1}$ is zero all along the curve $C$ and $f_{2}$ is zero except at those parts of $C$ which do not coincide with a characteristic. The solution in this case is $\psi \equiv 0$, except for an infinitesimally narrow strip which extends along the characteristic part of $C$. In this strip $\psi_{\rho}$ has the prescribed values. Since the width of this strip is infinitesimally small, an integration of $\psi_{\rho}$ across the strip will not give a change of $\psi$. The Fourier coefficients of such a "solution" are naturally zero. The boundary condition that $\psi_{\rho}$ has a jump which does not have the desired relation to the jump of $\psi$ is fulfilled by what is called a lost solution, i.e., a solution which cannot be obtained if one considers in the hodograph plane only those solutions for which the second derivatives of $\psi$ exist.

The simplifications which can be carried out in the expression for $a_{1}$ proceed in a similar manner. Application of Eq. (5.12) yields

$$
a_{1}=\frac{1}{2 \mu}\left\{\rho^{-1 / 12+\mu}\left(I_{\mathrm{I}}+I_{\mathrm{II}}\right)-\rho^{-1 / 12-\mu}\left(I_{\mathrm{III}}+I_{\mathrm{IV}}\right)\right\}
$$


where

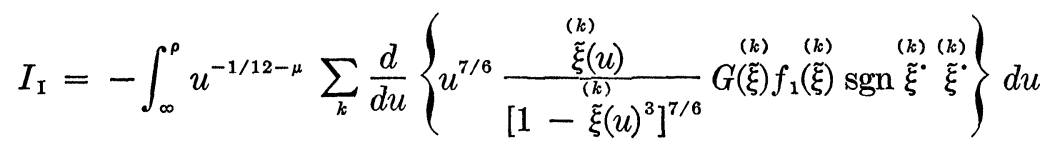

$$
\begin{aligned}
& I_{\mathrm{II}}=\int_{\infty}^{\rho} u^{-11 / 12-\mu} \sum_{k} \frac{\left(1-{\stackrel{(k)}{\xi})^{3 / 6}}^{5}\right.}{9} \operatorname{sgn} \stackrel{(k)}{\xi}^{\cdot}\left[-G^{(k)}(\tilde{\xi}) f_{1}^{\prime}(\tilde{\xi})+G^{\prime}(\stackrel{(k)}{\xi}) f_{1}^{(k)}(\tilde{\xi})\right] d u \\
& I_{\mathrm{III}}=-\int_{0}^{\rho} u^{-1 / 12+\mu} \sum_{k} \frac{d}{d u}\left\{u^{7 / 6} \frac{\stackrel{(k)}{\xi}(u)}{\left[1-{\left.\stackrel{(k)}{\xi}(u)^{3}\right]^{7 / 6}}_{(k)}^{(k)}\right.}\left(\stackrel{(k)}{\xi} f_{1}(\tilde{\xi}) \operatorname{sgn} \stackrel{(k)}{\xi}^{\cdot(k)} \tilde{\xi}^{\cdot}\right\} d u\right. \\
& I_{\mathrm{IV}}=\int_{0}^{\rho} u^{-11 / 12+\mu} \sum_{k} \frac{\left(1-\stackrel{(k)}{\xi}^{3}\right)^{5 / 6}}{9} \operatorname{sgn} \stackrel{(k)}{\tilde{\xi}^{\circ}}\left[-G^{(k)}(\tilde{\xi}) f_{1}^{\prime}(\tilde{\xi})+G^{\prime(\xi)}(\tilde{\xi}) f_{1}^{(k)}(\xi)\right] d u
\end{aligned}
$$

Consider first the integral $I_{\mathrm{I}}$. By carrying out the change of the variable of integration given by Eq. (5.14), one finds

$$
I_{\mathrm{I}}=\int_{\substack{-\infty \\ \tilde{\rho}>\rho}}^{1} \tilde{\rho}(v)^{-11 / 12-\mu} \frac{d}{d v}\left\{\tilde{\rho}(v)^{7 / 6} \frac{v}{\left(1-v^{3}\right)^{7 / 6}} G(v) f_{1}(v) \frac{1}{\tilde{\rho}^{\prime}(v)}\right\} d v
$$

Next an integration by parts is carried out. The upper and lower limits of the individual intervals of integrations are the values of $\stackrel{(k)}{\xi}$ for the particular value of $\rho$ considered. We have an upper limit if $\tilde{\xi}^{\cdot}$ or $\tilde{\rho}^{\prime}$ is negative, a lower limit if it is positive. At the limits of the intervals, one has $\tilde{\rho}=\rho$. Thus we find

$$
\begin{aligned}
& I_{1}=\sum_{k}- \rho^{13 / 12-\mu} \frac{\stackrel{(k)}{\xi}}{\left(1-\stackrel{(k)}{\xi}^{3}\right)^{7 / 6}} G(\tilde{\xi}) f_{1}(\tilde{\xi}) \frac{1}{(k)} \operatorname{sgn} \tilde{\rho}^{\prime} \\
& \tilde{\rho}^{\prime}(\tilde{\xi}) \\
&-\left(-\frac{1}{12}-\mu\right) \int_{\substack{-\infty \\
\tilde{\rho}>\rho}}^{1} \tilde{\rho}(v)^{1 / 12-\mu} \frac{v}{\left(1-v^{3}\right)^{7 / 6}} G(v, \mu) f_{1}(v) \frac{1}{\tilde{\rho}^{\prime}(v)} d v
\end{aligned}
$$

A similar transformation yields for $I_{\text {III }}$

$$
\begin{aligned}
I_{\mathrm{III}}=\sum_{k}-\rho^{13 / 12+\mu} \frac{\stackrel{(k)}{\tilde{\xi}}}{\left(1-\stackrel{(\tilde{\xi})}{\xi}^{3}\right)^{7 / 6}} G(\tilde{\xi}) f_{1}(\tilde{\xi}) \frac{1}{\tilde{\rho}^{\prime}(\tilde{\xi})} \operatorname{sgn} \tilde{\rho}^{\prime} \\
+\left(-\frac{1}{12}+\mu\right) \int_{\substack{-\infty \\
\tilde{\rho}<p}}^{1} \tilde{\rho}^{1 / 12+\mu} \frac{v}{\left(1-v^{3}\right)^{7 / 6}} G(v, \mu) f_{1}(v) \frac{1}{\tilde{\rho}^{\prime}(v)} d v
\end{aligned}
$$


In the combination $\rho^{-1 / 12+\mu} I_{\mathrm{I}}-\rho^{-1 / 12-\mu} I_{\mathrm{III}}$ which occurs in Eq. (5.17) the terms outside of the integrals drop out, and one finds

$$
\begin{aligned}
\rho^{-1 / 12-\mu} I_{\mathrm{I}} & -\rho^{-1 / 12-\mu} I_{\mathrm{III}} \\
= & -\left(-\frac{1}{12}-\mu\right) \rho^{-1 / 12+\mu} \int_{\substack{\tilde{\rho}>\rho \\
\rho}}^{1} \tilde{\rho}^{1 / 12-\mu} \frac{v}{\left(1-v^{3}\right)^{7 / 6}} G(v, \mu) f_{1}(v) \frac{1}{\tilde{\rho}^{\prime}(v)} d v \\
& -\left(-\frac{1}{12}+\mu\right) \rho^{-1 / 12-\mu} \int_{\substack{\tilde{\rho}<\infty \\
\tilde{\rho}<\rho}}^{1} \tilde{\rho}^{1 / 12+\mu} \frac{v}{\left(1-v^{3}\right)^{7 / 6}} G(v, \mu) f_{1}(v) \frac{1}{\tilde{\rho}^{\prime}(v)} d v
\end{aligned}
$$

Similar transformations are carried out in $I_{\mathrm{II}}$ and $I_{\mathrm{IV}}$

$$
\begin{aligned}
& I_{\mathrm{II}}=-\int_{\substack{\tilde{\rho} \boldsymbol{\rho} \\
\tilde{\rho}>\rho}}^{1} \tilde{\rho}(v)^{-11 / 12-\mu} \frac{\left(1-v^{3}\right)^{5 / 6}}{9}\left\{-G(v) f_{1}^{\prime}(v)+G^{\prime}(v) f_{1}(v)\right\} \tilde{\rho}^{\prime}(v) d v \\
& =\sum_{k} \frac{1}{\frac{1}{12}-\mu} \rho^{1 / 12-\mu} \frac{\left(1-{ }^{(k)} \xi^{3}\right)^{5 / 6}}{9}\left[-G^{(k)} \stackrel{(k)}{(\xi)} f_{1}^{\prime}(\xi)+G^{\prime}(\xi) \stackrel{(k)}{(\xi)} f_{1}(\xi)\right] \operatorname{sgn} \tilde{\rho}^{\prime} \\
& -\frac{1}{\frac{1}{12}-\mu} \int_{\substack{\tilde{c} \infty \\
\tilde{\rho}>\rho}}^{1} \tilde{\rho}(v)^{1 / 12-\mu} \frac{G(v)}{9} \frac{d}{d v}\left[\left(1-v^{3}\right)^{7 / 6} f_{1}^{\prime}(v)\right] d v \\
& +\left(-\mu-\frac{1}{12}\right) \int_{\substack{\tilde{\tilde{\rho}}>\rho \\
j}}^{1} \tilde{\rho}(v)^{1 / 12-\mu} \frac{v}{\left(1-v^{3}\right)^{7 / 6}} G(v) f_{1}(v) d v \\
& I_{\mathrm{III}}=\sum_{k} \frac{1}{\frac{1}{12}+\mu} \rho^{1 / 12+\mu} \frac{\left(1-\stackrel{(k)}{\xi}^{3}\right)^{5 / 6}}{9}\left[-G^{(\xi)}(\tilde{\xi}) f_{1}(\tilde{\xi})+G^{\prime(\tilde{\xi})}\left(\stackrel{(k)}{(\xi)} f_{1}(\tilde{\xi})\right] \operatorname{sgn} \tilde{\rho}^{\prime}\right. \\
& +\frac{1}{\frac{1}{12}+\mu} \int_{\substack{\tilde{\rho}<\rho \\
\rho}}^{1} \tilde{\rho}(v)^{1 / 12+\mu} \frac{G(v)}{9} \frac{d}{d v}\left[\left(1-v^{3}\right)^{7 / 6} f_{1}^{\prime}(v)\right] d v \\
& -\left(\mu-\frac{1}{12}\right) \int_{\substack{\tilde{\rho} \infty \\
\tilde{\rho}<\rho}}^{1} \tilde{\rho}(v)^{1 / 12+\mu} \frac{v}{\left(1-v^{3}\right)^{7 / 6}} G(v) f_{1}(v) d v
\end{aligned}
$$


Combining the expressions (5.21), (5.22), and (5.23), one obtains the final expression for $a_{1}$ from (5.17)

$$
\begin{gathered}
a_{1}(\rho)=\sum_{k} \frac{1}{\frac{1}{144}-\mu^{2}} \frac{\left(1-\stackrel{(k)}{\xi}^{3}\right)^{5 / 6}}{9}\left[-G\left(\stackrel{(k)}{(\xi)} f_{1}^{\prime}(\tilde{\xi})+G^{\prime}(\tilde{\xi}) f_{1}(\tilde{\xi})\right] \operatorname{sgn} \tilde{\rho}^{\prime}\right. \\
-\frac{1}{2 \mu}\left\{\frac{\rho^{-1 / 12+\mu}}{\frac{1}{12}-\mu} \int_{\substack{-\infty \\
\tilde{\rho}>\rho}}^{1} \tilde{\rho}(v)^{1 / 12-\mu} \frac{G(v)}{9} \frac{d}{d v}\left[\left(1-v^{3}\right)^{5 / 6} f_{1}^{\prime}(v)\right] d v\right. \\
\left.+\frac{\rho^{-1 / 12-\mu}}{\frac{1}{12}+\mu} \int_{\substack{-\infty \\
\tilde{\rho}<\rho}}^{1} \tilde{\rho}(v)^{1 / 12+\mu} \frac{G(v)}{9} \frac{d}{d v}\left[\left(1-v^{3}\right)^{5 / 6} f_{1}^{\prime}(v)\right] d v\right\}
\end{gathered}
$$

A very similar procedure applies to the imaginary values of $\mu$. The result is

$$
\begin{aligned}
a_{1}(\rho, \mu)=\sum_{k} & \frac{1}{\frac{1}{144}-\mu^{2}} \frac{\left(1-\stackrel{(k)}{\xi^{3}}\right)^{5 / 6}}{9}\left[-G(\tilde{\xi}) f_{1}^{\prime}(\tilde{\xi})+G^{\prime}(\tilde{\xi}) f_{1}(\tilde{\xi})\right] \operatorname{sgn} \tilde{\rho}^{\prime} \\
& -\operatorname{Re}\left\{\frac{\rho^{-1 / 12+\mu}}{\mu\left(\frac{1}{12}-\mu\right)} \int_{\substack{-\infty \\
\tilde{\rho}>\rho}}^{1} \tilde{\rho}^{1 / 12-\mu} \frac{G(v)}{9} \frac{d}{d v}\left[\left(1-v^{3}\right)^{5 / 6} f_{1}^{\prime}(v)\right] d v\right\}
\end{aligned}
$$

In this derivation it has been tacitly assumed that all differentiations required can be carried out, in particular that $f_{1}$ can be differentiated twice.

The solution of the inhomogeneous boundary value problem is now found by inserting the expressions for $a_{3}, a_{2}$ and $a_{1}$ found in Eqs. (5.11), (5.15), (5.16), (5.24), and (5.25) into Eq. (5.10) and then (5.10) into the original hypothesis (5.8). The result is simplified by the fact that in Eq. (5.8) the terms due to $a_{3}$ cancel the term $\chi$.

\section{Transformation of the Solution of the Inhomogeneous Boundary Value Problem}

It will be the aim of the subsequent investigations to present the solution of the previous boundary value problem in the form of a superposition of the particular solutions of the first family. As it stands, the solution (5.8) uses functions $G$ of the second family, but even the particular solutions (2.5) which are formed with these functions are not evident, since in the integrals which appear in (5.2) and elsewhere the limits depend upon $\rho$.

The last term of Eq. (5.8) requires an integration along the imaginary axis of the complex $\mu$-plane. The desired transformation of the solution is carried out by deforming this path of integration. The functions which occur in the integral must be shown to be analytic functions of $\mu$, and furthermore the conditions under which such a deformation is admissible must be investigated. 
In Section 3, the shape of the region in which an infinite series of the first family of particular solutions would converge has been determined. It follows from these investigations that no region of convergence exists unless $f_{1}, f_{2}$ and $\tilde{\rho}$ are free of singularities in the vicinity of $\xi=1$. Thus it is in the nature of the present problem to introduce the requirement that $f_{1}, f_{2}$ and $\tilde{\rho}$ are analytic functions of $\xi$ in the vicinity of $\xi=1$, say for $\xi_{1}<\xi<1$, where $\xi_{1}$ is a suitable constant. To assume that all derivatives of these functions exist for real values of $\xi$ is not sufficient for the following investigations.

For $-\infty<\xi<\xi_{1}$ the functions $f_{1}, f_{2}$ and $\tilde{\rho}$ need to be defined for real values of $\xi$ only. Naturally it is assumed that the derivatives which occur in the expressions (5.15), (5.16), (5.24) and (5.25) exist. In these formulæ a variable $v$ appears instead of $\xi$ as argument of these functions. In the integrals from $v=-\infty$ to $v=1$ which occur in these expressions, the intervals from $-\infty$ to $\xi_{1}$ and from $\xi_{1}$ to 1 will be treated separately. The contribution to $\psi$ due to the intervals will be denoted by $\psi_{\mathrm{I}}$ and $\psi_{\mathrm{II}}$ respectively. Thus we have

$$
\psi=\psi_{\mathrm{I}}+\psi_{\mathrm{II}}
$$

One will notice that $\psi_{\mathrm{I}}$ is not the solution of an inhomogeneous boundary value problem for which in the interval $-\infty<\xi<\xi_{1}$ the jump of $\psi$ is given by $f_{1}$ and the jump of $\psi_{\rho}$ is given by $f_{2}$, while for $\xi_{1}<\xi<1$ the jump of these quantities is zero, for functions so defined would not be differentiable at $\xi=\xi_{1}$. The same holds for $\psi_{\mathrm{II}}$, but not for $\psi=\psi_{\mathrm{I}}+\psi_{\mathrm{II}}$. The following assumptions about $\rho$ will be made:

$\rho$ is so small that $\tilde{\xi}(\rho)$ is one valued and that $\xi_{1}<\tilde{\xi}(\rho)<1$. The curve $C$ may be of such a nature that in the interval $-\infty<\xi<\xi_{1}$, one has $\tilde{\rho}\left(\xi_{1}\right) \leqq \tilde{\rho}(\xi)$. If the curve $C$ intersects in the $\eta, \theta$-plane the line $\xi=1$ at a finite distance from the origin, one finds that $\rho \rightarrow 0$ if $\xi \rightarrow 1$; therefore $\tilde{\rho}^{\prime}$ is negative for this choice of $\rho$. The functions $\tilde{\rho}, f_{1}$ and $f_{2}$ and their derivatives are assumed to be bounded, where necessary.

For simplicity, set

and

$$
f_{3}(v)=\frac{1}{9} \frac{d}{d v}\left[\left(1-v^{3}\right)^{5 / 6} f_{1}^{\prime}(v)\right]
$$

$$
f_{4}(v)=f_{2}(v)\left[1-\frac{\left(1-v^{3}\right)^{2}}{9 v} \frac{\tilde{\rho}^{\prime}(v)^{2}}{\tilde{\rho}(v)^{2}}\right] \tilde{\rho}(v) \frac{v}{\left(1-v^{3}\right)^{7 / 6}}
$$

The function $\psi_{\mathrm{I}}$ is then obtained by inserting Eqs. (5.11), (5.15), (5.16), (5.24), and (5.25) into (5.8).

$$
\begin{gathered}
\psi_{\mathrm{I}}=\frac{3}{\pi}\left\{\sum_{\mu_{h}} \frac{G\left(\xi, \mu_{h}\right) \rho^{-1 / 12+\mu_{h}}}{B\left(\mu_{h}\right) B\left(-\mu_{h}\right) 2 \mu_{h}} \int_{-\infty}^{\xi_{1}} \tilde{\rho}(v)^{1 / 12-\mu_{h}} G\left(v, \mu_{h}\right)\left[f_{4}(v)+\frac{f_{3}(v)}{\mu_{h}-\frac{1}{12}}\right] d v\right. \\
-\operatorname{Re} \int_{\mu=0}^{i \infty}\left\{\frac{i G(\xi, \mu)}{B(\mu) B(-\mu) \sin \left[\pi\left(\mu+\frac{1}{4}\right)\right] \sin \left[\pi\left(-\mu+\frac{1}{4}\right)\right]} \frac{\rho^{-1 / 12+\mu}}{2 \mu}\right. \\
\left.\cdot \int_{-\infty}^{\xi_{1}} \tilde{\rho}(v)^{1 / 12-\mu} G(v, \mu)\left[f_{4}(v)+\frac{f_{3}(v)}{\mu-\frac{1}{12}}\right] d v\right\} d \mu
\end{gathered}
$$


In the integrals over $v$ the limits are fixed because of the assumption made above for the choice of $\rho$. If $\rho$ and $\xi$ are considered as fixed, the second term in Eq. (7.3) is the integral over an analytic function of $\mu$. This is obvious for all expressions of the integrand except for the integral over $v$. In this integral $f_{3}, f_{4}$ and $\tilde{\rho}$ are bounded; $G$ is bounded, too, since the interval of integration does not extend to the point $v=1$. Furthermore, $G$ behaves for the vicinity of $\xi=-\infty$ as $(-\xi)^{-\frac{3}{2}}$; thus, the integral converges uniformly for any finite region of the complex $\mu$-plane which does not contain the point $\mu=1 / 12$ and defines an analytic function.

It will be shown later that for large absolute values of $\mu$ the integrand has such properties that one can deform the path of integration from the imaginary axis of the complex $\mu$-plane to the real axis. The integrand has no poles in the first quadrant of the complex $\mu$-plane except along the real axis. The path of integration will follow the real axis except for indentations in the vicinity of the poles. Now, the integrand of the second term in (7.3) is imaginary along the real $\mu$-axis, since for real values of $\mu$ the function $G$ and all other functions occurring in the integrand are real. Thus the only contribution to the real part is due to the indentations which exclude the poles; for a given pole, it is given by the residue of this pole multiplied by $-i \pi$. The residues of all the poles are imaginary; thus, the contributions of the indentations are real. By this process the second term in Eq. (7.3) will finally appear as an infinite sum, $c f$. Fig. 5.

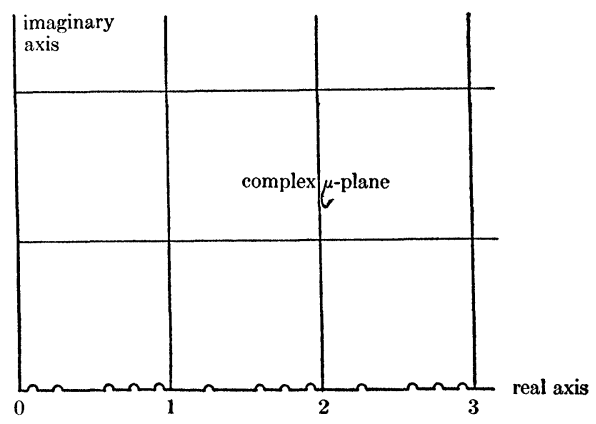

FIG. 5.

Thus, we have to carry out the following program:

1. Determination of the poles and of their residues.

2. Investigation of the behavior of the integrand for $|\mu| \rightarrow \infty$, in order to justify the deformation of the path of integration.

As mentioned above, one pole arises at $\mu=1 / 12$ because of the term with $f_{3}$. Its residue is immediately obvious as

$$
\frac{i G\left(\xi, \frac{1}{12}\right)}{B\left(\frac{1}{12}\right) B\left(-\frac{1}{12}\right) \sin \frac{\pi}{3} \sin \frac{\pi}{6}} \frac{1}{\frac{1}{6}} \int_{-\infty}^{\xi_{2}} G\left(v, \frac{1}{12}\right) f_{3}(v) d v
$$


By means of Eqs. (7.2), (1.7) and (1.9) this expression can be simplified to give

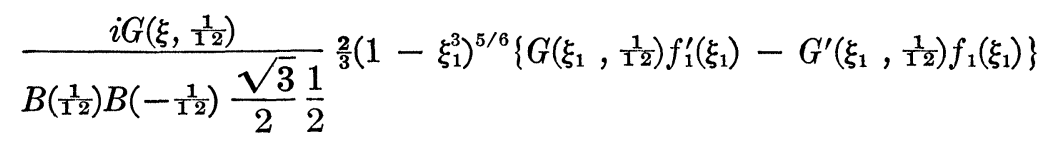

All other poles arise because of the factor

$$
\left\{B(\mu) B(-\mu) \sin \left[\pi\left(\mu+\frac{1}{4}\right)\right] \sin \left[\pi\left(-\mu+\frac{1}{4}\right)\right] 2 \mu\right\}^{-1}
$$

occurring in the integrand. Let us consider first the poles at the points $\mu=h-\frac{1}{4}$, which are caused by the term $\sin \left[\pi\left(\mu+\frac{1}{4}\right)\right]$. These are the quantities $\mu_{h}$ occurring in the first term of Eq. (7.3). The residues at these points are found to be

$$
\frac{i G\left(\xi, \mu_{h}\right) \rho^{-1 / 12+\mu_{h}}}{B\left(\mu_{h}\right) B\left(-\mu_{h}\right) \pi 2 \mu_{h}} \int_{-\infty}^{\xi_{1}} \tilde{\rho}(v)^{1 / 12-\mu_{h}} G\left(v, \mu_{h}\right)\left(f_{4}(v)+\frac{f_{3}(v)}{\mu_{h}+\frac{1}{12}}\right) d v
$$

Multiplying this by $-i \pi$ in order to obtain the contribution of these poles to the second term in (7.3), one finds that these expressions cancel the first sum in the equation (7.3).

To determine the residues of the remaining poles, we put these poles in evidence by means of Eq. (4.5) (which defines the functions $B$ ) and by some familiar relations for the $\Gamma$-functions

$$
\begin{aligned}
& \frac{1}{B(\mu) B(-\mu) \sin \left[\pi\left(-\mu+\frac{1}{4}\right)\right] 2 \mu} \\
& \quad=\frac{\Gamma\left(\mu+\frac{11}{12}\right) \Gamma\left(\mu+\frac{7}{12}\right) \Gamma\left(-\mu+\frac{11}{12}\right) \Gamma\left(-\mu+\frac{7}{12}\right)}{\Gamma(2 \mu) \Gamma(-2 \mu) 2 \mu \sin \left[\pi\left(-\mu+\frac{1}{4}\right)\right]} \\
& \quad=\frac{\Gamma\left(\mu+\frac{1}{12}\right) \Gamma\left(\mu+\frac{7}{12}\right) \pi \sin 2 \pi \mu}{\Gamma\left(\mu+\frac{1}{12}\right) \Gamma\left(\mu+\frac{5}{12}\right) \sin \left[\pi\left(\mu+\frac{1}{12}\right)\right] \sin \left[\pi\left(\mu+\frac{5}{12}\right)\right] \sin \left[\pi\left(\mu-\frac{1}{4}\right)\right]}
\end{aligned}
$$

Poles arise at the points where the sine functions vanish, i.e., at the points $\mu=\frac{1}{4}+h / 3$. These are exactly the values of $\mu$ occurring in the first family of particular solutions. The residues can then be evaluated by a direct computation. Since the procedure is straightforward, we give only the resulting expression for $\psi_{\mathrm{I}}$, which includes naturally also the contribution of the point $\mu=\frac{1}{12}$

$$
\begin{aligned}
\psi_{\mathrm{I}}=\frac{3}{\pi} & \left\{\frac{-\pi G\left(\xi, \frac{1}{12}\right) 8}{B\left(\frac{1}{12}\right) B\left(-\frac{1}{12}\right) 3 \sqrt{3}}\left(1-\xi_{1}^{3}\right)^{5 / 6}\left[G\left(\xi_{1}, \frac{1}{12}\right) f_{1}^{\prime}\left(\xi_{1}\right)-G^{\prime}\left(\xi_{1}, \frac{1}{12}\right) f_{1}\left(\xi_{1}\right)\right]\right. \\
& -\sum_{h=0}^{\infty} \frac{\Gamma\left(\frac{h}{3}+\frac{7}{6}\right) \Gamma\left(\frac{h}{3}+\frac{5}{6}\right)}{\Gamma\left(\frac{h}{3}+\frac{1}{3}\right) \Gamma\left(\frac{h}{3}+\frac{2}{3}\right)} \frac{4}{3} G\left(\xi, \frac{h}{3}+\frac{1}{4}\right) \rho^{1 / 6+h / 3} \\
& \left.\cdot \int_{-\infty}^{\xi_{1}} \tilde{\rho}(v)^{-1 / 6-h / 3} G\left(v, \frac{h}{3}+\frac{1}{4}\right)\left[f_{3}(v)+\frac{f_{4}(\nu)}{\frac{h}{3}+\frac{1}{6}}\right] d v\right\}
\end{aligned}
$$


The infinite series occurring here has the expected form. The first term, however, would be singular at the characteristic $\xi=1$. Later it will cancel an expression occurring in $\psi_{\mathrm{II}}$.

To justify the deformation of the path of integration from the imaginary to the real $\mu$-axis, we make use of a family of contours $C_{n}$ given by circles $|\mu|=$ const. which connect the imaginary axis with the real axis. Their radii $|\mu|$ are chosen in such a manner that $|\mu| \rightarrow \infty$ as $n \rightarrow \infty$ and that the minimum distance of the curve $C_{n}$ from the poles always exceeds a fixed positive quantity.

According to the assumptions made previously, the smallest value of $\tilde{\rho}$ which occurs in the interval $-\infty<\xi<\xi_{1}$, is found at $\xi=\xi_{1}$. An estimate for $G$ is given by Eq. (1.16); $f_{1}$ and $f_{2}$, and also $f_{3}$ and $f_{4}$ are supposed to be bounded. Thus

$$
\int_{-\infty}^{\xi_{1}} \tilde{\rho}^{-1 / 12-\mu} G(v, \mu)\left[f_{3}(v)+\frac{f_{4}(v)}{\mu-\frac{1}{12}}\right] d v<M \frac{e^{\pi \operatorname{Im} \mu}}{\mu}\left[\frac{1+\xi_{1}^{3 / 2}}{1-\xi_{1}^{3 / 2}} \cdot \frac{1}{\tilde{\rho}\left(\xi_{1}\right)}\right]^{\mu}
$$

where $M$ is a finite quantity independent of $\mu$.

From Eq. (7.4) and by means of StirLing's formula one finds

$$
\begin{aligned}
& \left|\frac{1}{B(\mu) B(-\mu) 2 \mu \sin \left[\pi\left(\mu+\frac{1}{4}\right)\right] \sin \left[\pi\left(-\mu+\frac{1}{4}\right)\right]}\right| \\
& <M \frac{e^{2 \pi \operatorname{Im} \mu}}{\sin \left[\pi\left(\mu+\frac{11}{12}\right)\right] \sin \left[\pi\left(\mu+\frac{5}{12}\right] \sin \left[\pi\left(\mu+\frac{1}{4}\right)\right] \sin \cdot\left[\pi\left(-\mu+\frac{1}{4}\right)\right]\right.}
\end{aligned}
$$

The contours $C_{n}$ considered avoid the immediate vicinity of the poles; one therefore finds

$$
\frac{1}{B(\mu) B(-\mu) 2 \mu \sin \left[\pi\left(\mu+\frac{1}{4}\right)\right] \sin \left[\pi\left(-\mu+\frac{1}{4}\right)\right]}<M \mu e^{-2 \pi \operatorname{Im} \mu}
$$

for the contours $C_{n}$.

Thus the following estimation for the integrand of the second term in Eq. (7.3) is obtained

$$
M e^{-\pi \operatorname{Im} \mu}\left[\frac{1+\xi_{1}^{\frac{3}{2}}}{1-\xi_{1}^{\frac{3}{2}}} \frac{1}{\tilde{\rho}\left(\xi_{1}\right)}\right]^{\mu} G(\xi, \mu) \rho^{\mu}
$$

Now, asymptotic expressions for the function $G(\xi, \mu)$ are inserted. For $\xi<0$ one finds by means of Eq. (1.16a)

$$
M \frac{1}{\mu} e^{-\pi \operatorname{Im} \mu} \sin \left[2 \mu\left(\frac{\pi}{2}-\xi^{\prime}\right)\right]\left[\frac{1+\xi_{1}^{\frac{3}{2}}}{1-\xi_{1}^{\frac{3}{2}}} \frac{\rho}{\tilde{\rho}\left(\xi_{1}\right)}\right]^{\mu}
$$

The largest possible value of $\left(\frac{1}{2} \pi-\xi^{\prime}\right)$ is $\frac{1}{2} \pi$ and thus $e^{-\pi \operatorname{Im} \mu} \sin \left[2 \mu\left(\frac{1}{2} \pi-\xi^{\prime}\right)\right]$ is of $O(1)$. This gives as final upper bound for the integrand

valid for $\xi<0$.

$$
M \frac{1}{\mu}\left[\frac{1+\xi_{1}^{\frac{3}{2}}}{1-\xi_{1}^{\frac{3}{2}}} \frac{\rho}{\rho\left(\xi_{1}\right)}\right]^{\mu}
$$


For $\xi>0$ one finds in a similar manner by means of (1.16b) as an estimate for the integrand

$$
M \frac{1}{\mu}\left[\frac{1+\xi_{1}^{\frac{3}{2}}}{1-\xi_{1}^{\frac{3}{3}}} \frac{1+\xi^{\frac{3}{2}}}{1-\xi^{\frac{3}{2}}} \frac{\rho}{\tilde{\rho}\left(\xi_{1}\right)}\right]^{\mu}
$$

Now it can be shown (e.g. Ref. 10, Chapter 6) that the integral under consideration extended along the contours $C_{n}$ vanishes for $n \rightarrow \infty$ if

$$
\frac{1+\xi_{1}^{\frac{3}{2}}}{1-\xi_{1}^{\frac{3}{2}}} \frac{\rho}{\rho\left(\xi_{1}\right)}<1 \text { for } \xi<0
$$

and if

$$
\frac{1+\xi_{1}^{\frac{3}{2}}}{1-\xi_{1}^{\frac{3}{2}}} \frac{1+\xi^{\frac{3}{2}}}{1-\xi^{\frac{3}{2}}} \frac{\rho}{\rho\left(\xi_{1}\right)}<1 \text { for } \xi>0
$$

For an interpretation of the first condition, we write it as

$$
\rho<\tilde{\rho}\left(\xi_{1}\right) \frac{1-\xi_{1}^{\frac{3}{2}}}{1+\xi_{1}^{\frac{3}{2}}}
$$

and express $\tilde{\rho}\left(\xi_{1}\right)$ and $\xi_{1}$ by means of $\eta_{1}$ and $\theta_{1}$ which denote the values of $\eta$ and $\theta$ at the point $\xi_{1}$ of the curve $C$.

$$
\rho<\left(\frac{3}{2} \theta_{1}-\eta_{1}^{\frac{3}{2}}\right)^{2} \text { for } \xi<0
$$

Similarly one obtains from Inequality $(7.6 \mathrm{~b})$

$$
\frac{3}{2} \theta+\eta^{\frac{3}{2}}<\frac{3}{2} \theta_{1}-\eta_{1}^{\frac{3}{2}}
$$

The expressions on the right hand sides are constant along characteristics with a positive slope. The expression on the left hand side of $(7.7 \mathrm{~b})$ is constant along a characteristic with a negative slope. Thus one arrives at the following construction for the region in which the deformation of the contour is allowed (Fig. 6). One draws through the point $\xi_{1}$ of the curve $C$ the characteristic which

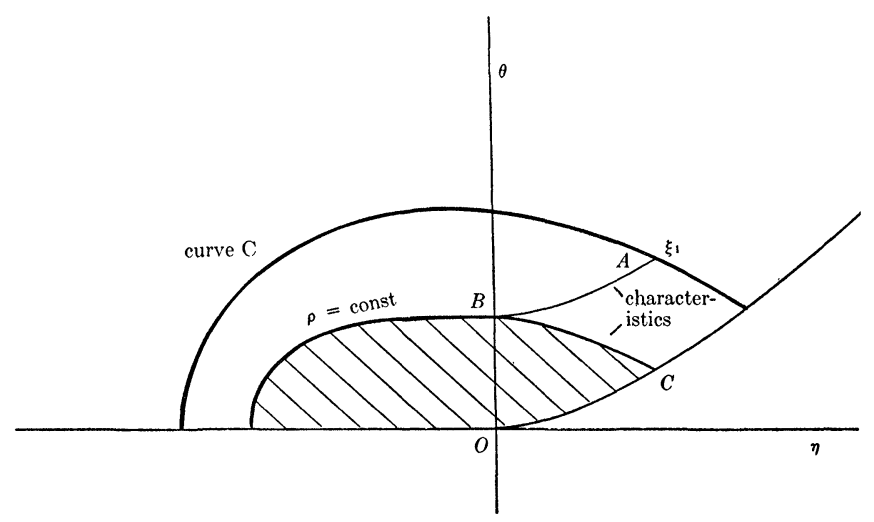

FIG. 6. 
has a positive slope. Through its point of intersection with the line $\eta=0$, one draws the line $\rho=$ const. and the characteristic which has a negative slope. The shaded region is then the region in which the deformation of the path of integration is justified. Obviously this region is exactly the region in which one can expect convergence of the series, for it is not excluded by our assumptions that a singularity propagates along the characteristic $A B$ (Fig. 6). If this is the case, it would then be reflected at the sonic line and propagate along $B C$. Since a development with respect to particular solutions of the first family represents along a line $\xi=$ const. a power series in $\rho^{\frac{1}{3}}$, the region of convergence cannot be expected to extend beyond the characteristic $B C$.

In the treatment of $\psi_{\mathrm{Ir}}$, the previous discussions must be supplemented by additional considerations, because of the following reasons:

1. The limits of the integrals occurring in Eqs. (5.15), (5.16), (5.24) and (5.25) depend here upon the value of $\rho$.

2. The integral over $v$ in general will not converge if the real part of $\mu$ exceeds a certain limit. Such an integral will occur, however, if a deformation of the contour of integration in the complex $\mu$-plane similar to that in the treatment of $\psi_{\mathrm{I}}$ is carried out. Therefore, the integrals over $v$ must be redefined in such a manner that their analytic continuation through the entire $\mu$-plane is possible. Here the assumption that $f_{1}, f_{2}$, and $\tilde{\rho}$ are analytic functions is of importance.

Expressing the function $G$ which occurs in the integral over $v$, by means of the function $G_{1}$ defined in Eq. (1.17), one finds the following expression for $\psi_{\mathrm{II}}$

$$
\psi_{\mathrm{II}}=\frac{3}{\pi}\left[E_{1}+E_{2}+E_{3}+E_{4}+E_{5}+E_{6}\right]
$$

where

$$
\begin{gathered}
E_{1}=\sum_{h} \frac{G\left(\xi, \mu_{h}\right)(-)^{h}}{\left(\frac{1}{144}-\mu_{h}^{2}\right) g B\left(\mu_{h}\right)}\left[1-\tilde{\xi}^{3}(\rho)\right]^{5 / 6}\left[G_{1}\left(\xi(\rho), \mu_{h}\right)\right. \\
\left.\cdot f_{1}^{\prime}(\tilde{\xi}(\rho))-G_{1}^{\prime}\left(\tilde{\xi}(\rho), \mu_{h}\right) f_{1}(\xi(\rho))\right] \\
E_{2}=\operatorname{Re} \int_{0}^{i \infty}-G(\xi, \mu) \frac{i d \mu\left[1-\tilde{\xi}(\rho)^{3}\right]^{5 / 6}\left[G_{1}(\tilde{\xi}(\rho), \mu) f_{1}^{\prime}(\tilde{\xi}(\rho))-G_{1}^{\prime}(\tilde{\xi}(\rho), \mu) f_{1}(\xi(\rho))\right]}{\left(\frac{1}{144}-\mu^{2}\right) 9 B(\mu) \sin \left[\pi\left(\mu+\frac{1}{4}\right)\right]} \\
E_{3}=\sum_{h} \frac{G\left(\xi, \mu_{h}\right)(-)^{h}}{2 \mu_{h} B\left(\mu_{h}\right)} \int_{\xi}^{\xi(\rho)}\left(\frac{\rho}{\tilde{\rho}(v)}\right)^{-1 / 12+\mu_{h}} G_{1}\left(v, \mu_{h}\right)\left[\frac{f_{3}(v)}{\mu_{h}-\frac{1}{12}}+f_{4}(v)\right] d v \\
E_{4}=\sum_{h} \frac{G\left(\xi, \mu_{h}\right)(-)^{h}}{2 \mu_{h} B\left(\mu_{h}\right)} \int_{\tilde{\xi}(\rho)}^{1}\left(\frac{\rho}{\tilde{\rho}(v)}\right)^{-1 / 12-\mu_{h}} G_{1}\left(v, \mu_{h}\right)\left[\frac{f_{3}(v)}{-\mu_{h}-\frac{1}{12}}+f_{4}(v)\right] d v \quad(7.9 \mathrm{~d})
\end{gathered}
$$




$$
\begin{gathered}
E_{5}=\operatorname{Re} \int_{\mu=0}^{i \infty} \frac{-i d \mu G(\xi, \mu)}{2 \mu B(\mu) \sin \left[\pi\left(\mu+\frac{1}{4}\right)\right]} \int_{\xi_{1}}^{\tilde{\xi}(\rho)}\left(\frac{\rho}{\tilde{\rho}(v)}\right)^{-1 / 12+\mu} G_{1}(v, \mu)\left[\frac{f_{3}(v)}{\mu-\frac{1}{12}}+f_{4}(v)\right] d v \\
E_{6}=\operatorname{Re} \int_{\mu=0}^{i \infty} \frac{-i d \mu G(\xi, \mu)}{2 \mu B(-\mu) \sin \left[\pi\left(-\mu+\frac{1}{4}\right)\right]} \\
\cdot \int_{\xi_{1}}^{\xi(\rho)}\left(\frac{\rho}{\tilde{\rho}(v)}\right)^{-1 / 12+\mu} G_{1}(v,-\mu)\left[\frac{f_{3}(v)}{\mu-\frac{1}{12}}+f_{4}(v)\right] d v
\end{gathered}
$$

If in the expression $E_{2}$ the integration is carried out along the real instead of along the imaginary axis one obtains, except for the one term which is caused by the residue at $\mu=1 / 12$, the expression $-E_{1}$. One thus finds

$$
E_{1}+E_{2}=\frac{2}{3} \pi \frac{G\left(\xi, \frac{1}{12}\right)\left(1-\xi^{3}\right)^{5 / 6}\left[G_{1}(\xi(\rho)) f_{1}^{\prime}(\xi(\rho))-G_{1}^{\prime}(\xi(\rho)) f_{1}(\xi(\rho))\right]}{B\left(\frac{1}{12}\right) \sin \frac{\pi}{3}}
$$

In the expression $E_{5}$, the path of integration can be deformed into the real axis under conditions which are much less restrictive than those found previously. Residues lie at $\mu=1 / 12$ and at the zeros of $\sin \left[\pi\left(\mu+\frac{1}{4}\right)\right]$. These residues cancel the term $E_{3}$. One thus obtains

$$
E_{3}+E_{5}=-\frac{6 G\left(\xi, \frac{1}{12}\right) \pi}{B\left(\frac{1}{12}\right) \sin \frac{\pi}{3}} \int_{\xi_{1}}^{\xi(\rho)} G_{1}\left(v, \frac{1}{12}\right) f_{3}(v) d v
$$

The function $G_{1}$ (Eq. (1.17)) satisfies the differential equation for $G$, and thus calculations similar to those which led to the expression (7.4a) can be carried out:

$$
E_{3}+E_{5}=-\left.\frac{2}{3} \frac{G\left(\xi, \frac{1}{12}\right)}{B\left(\frac{1}{12}\right) \sin \frac{\pi}{3}}\left[G_{1}\left(v, \frac{1}{12}\right) f_{1}^{\prime}(v)-G_{1}^{\prime}\left(v, \frac{1}{12}\right) f_{1}(v)\right]\right|_{\xi_{1}} ^{\xi(\rho)}
$$

This expression can be combined with Eq. (7.10) and one finds

$$
E_{1}+E_{2}+E_{3}+E_{5}=\frac{2 \pi G\left(\xi, \frac{1}{12}\right)}{3 B\left(\frac{1}{12}\right) \sin \frac{\pi}{3}}\left[G_{1}\left(\xi, \frac{1}{12}\right) f_{1}^{\prime}\left(\xi_{1}\right)-G_{1}^{\prime}\left(\xi, \frac{1}{12}\right) f_{1}\left(\xi_{1}\right)\right]
$$

$E_{4}$ can be written as a line integral which extends along the real $\mu$-axis with indentations which exclude the poles of the integrand. These indentations may lie below the real $\mu$-axis (Fig. 7)

$$
\begin{aligned}
& E_{4}=\operatorname{Re} \int_{0}^{\infty}-\frac{i d \mu G(\xi, \mu)}{2 \mu B(\mu) \sin \left[\pi\left(\mu+\frac{1}{4}\right)\right]} \\
& \cdot \int_{\tilde{\xi}(\rho)}^{1}\left(\frac{\rho}{\tilde{\rho}(v)}\right)^{-1 / 12-\mu} G_{1}(v, \mu)\left[\frac{f_{3}(v)}{-\mu-\frac{1}{12}}+f_{4}(v)\right] d v
\end{aligned}
$$




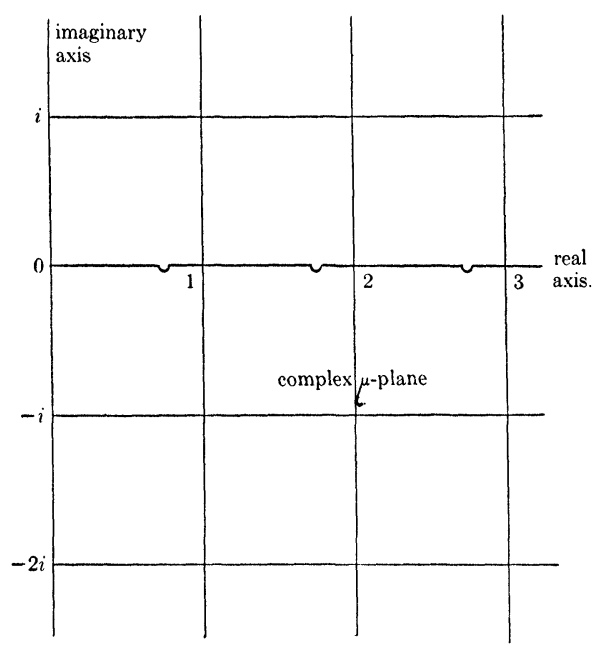

FIG. 7.

This path of integration will be deformed into the negative imaginary axis and then $\mu$ will be replaced consistently by $-\mu$. After this process $E_{4}$ will be combined with $E_{6}$

$$
E_{4}+E_{6}=\operatorname{Re} \int_{0}^{i \infty} \frac{-i d \mu G(\xi, \mu) \rho^{-1 / 12+\mu}}{2 \mu B(-\mu) \sin \left[\pi\left(-\mu+\frac{1}{4}\right)\right]} J(\mu)
$$

where

$$
J(\mu)=\int_{\xi_{1}}^{1} \tilde{\rho}(v)^{\mu-1 / 12} G_{1}(v,-\mu)\left[\frac{f_{3}(v)}{\mu-\frac{1}{12}}+f_{4}(v)\right] d v
$$

By the steps carried out so far, the dependence of the limit of the integral over $v$ on $\rho$ has dropped out. Now one must carry out the deformation of the path of integration from the imaginary to the $\mu$-axis. Here the difficulty mentioned above, that the integral will not converge if the real part of $\mu$ exceeds a certain value, will occur.

We now assume that $\tilde{\rho}, f_{3}$ and $f_{4}$ can be expressed in the following form

$$
\begin{aligned}
\tilde{\rho}(v) & =\left(1-v^{3}\right)^{\alpha_{1}} P_{1}\left(1-v^{3}\right) \\
f_{3}(v) & =\left(1-v^{3}\right)^{\alpha_{2}} P_{2}\left(1-v^{3}\right) \\
f_{4}(v) & =\left(1-v^{3}\right)^{\alpha_{2}} P_{3}\left(1-v^{3}\right)
\end{aligned}
$$

where $P_{1}, P_{2}$ etc. denote power series with a radius of convergence different from zero. $\alpha_{1}$ and $\alpha_{2}$ are in general real. One notices that the exponent $\alpha_{2}$ is the same for $f_{3}$ and $f_{4}$. If this is not the case or if $f_{3}$ and $f_{4}$ are the sums of several terms of the form (7.13) but with different values of $\alpha_{2}$, one must treat these expressions separately. In this case we should assume that only a finite number of such expressions are present. Now $J$ can be written as 


$$
J(\mu)=\int_{\xi_{1}}^{1}\left(1-v^{3}\right)^{\left(\alpha_{1}+1\right)(1 / 12-\mu)+\alpha_{2}} P_{4}\left(1-v^{3}\right) d v
$$

where the power series $P_{4}$ arises by a combination of the other power series. In the complex $\mu$-plane this integral converges at $\xi=1$ for an imaginary value of $\mu$, if $f_{1}, f_{2}$ and $\tilde{\rho}$ are suitably given. The integral can be rewritten in the form

$$
J(\mu)=\left\{1-e^{\left.-2 i \pi_{[}\left(\alpha_{1}+1\right)(1 / 12-\mu)+\alpha_{2}\right]}\right\}^{-1} \int_{C^{\prime}}\left(1-v^{3}\right)^{\left(\alpha_{1}+1\right)(1 / 12-\mu)+\alpha_{2}} P_{4}\left(1-v^{3}\right) d v
$$

where $C^{\prime}$ is the contour shown in Figure 8, and where along the upper part of $C^{\prime}$ the principal value of the integrand is used. This is correct for values of $\mu$ for which the integral (7.14) converges, for then the circle around the point $\xi=1$ can be contracted into this point, and one obtains merely the integral to be taken once from $\xi_{1}$ to 1 and a second time from 1 to $\xi_{1}$. These paths lie

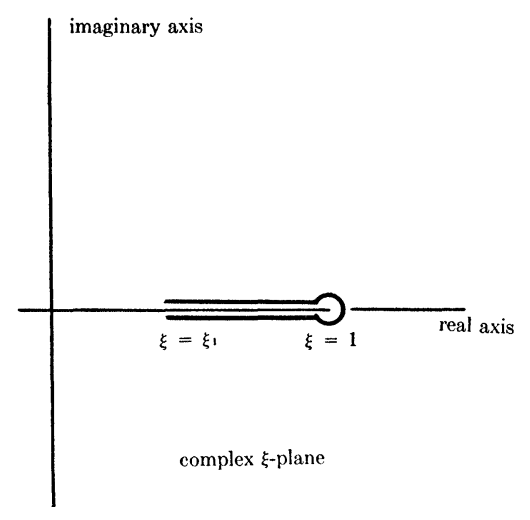

FIG. 8.

on different Riemann sheets and therefore the integrands differ by a complex factor. This factor is cancelled by the expression in front of the integral. Since the integrand is bounded along $C^{\prime}$ in any finite region of the complex $\mu$-plane, except for the obvious pole at $\mu=1 / 12$, the integral converges uniformly and thus provides the analytic continuation of the expression (7.14) throughout the entire $\mu$-plane. The function $J$ has poles along the real $\mu$-axis at those points for which the denominator of the factor in front vanishes unless the integral vanishes simultaneously. The significance of these poles will be discussed later. Going back to the original notations, we have now as representation for the integral $J$ the following expression

$$
J(\mu)=\left\{1-e^{\left.-2 i \pi_{I}\left(\alpha_{1}+1\right)(1 / 12-\mu)+\alpha_{2}\right]}\right\}^{-1} \int_{C^{\prime}} \tilde{\rho}(v)^{1 / 12-\mu} G_{1}(v,-\mu)\left[\frac{f_{3}(v)}{\mu-\frac{1}{12}}+f_{4}(v)\right] d v
$$


For the particular solutions of the first family, i.e., for $\mu=\frac{1}{4}+h / 3, J$ can be written in a different form. In general $G(\xi, \mu)$ is a linear combination of the functions $G_{1}(\xi, \mu)$ and $G_{1}(\xi,-\mu)$ as can be seen from Eqs. (1.10d) and (1.17). For these values of $\mu$ the coefficient of $G_{1}(\xi, \mu)$ in Eq. (1.10b) is zero and one finds

$$
G(\xi, \mu)=\Gamma\left(\frac{1}{2}\right) \frac{\Gamma\left(\frac{1}{2}+\frac{2 h}{3}\right) \sin \left[\pi\left(\frac{1}{2}+\frac{h}{3}\right)\right.}{\Gamma\left(\frac{7}{6}+\frac{h}{3}\right) \Gamma\left(\frac{5}{6}+\frac{h}{3}\right)} G_{1}(\xi,-\mu)
$$

Thus

$$
\begin{aligned}
J\left(\frac{1}{4}+\frac{h}{3}\right)=\{1- & \left.e^{\left.-2 i \pi_{1}\left(1+\alpha_{1}\right)(-1 / 6-h / 3)+\alpha_{2}\right]}\right\}^{-1} \\
& \cdot \frac{\Gamma\left(\frac{7}{6}+\frac{h}{3}\right) \Gamma\left(\frac{5}{6}+\frac{h}{3}\right)}{\Gamma\left(\frac{1}{2}+\frac{2 h}{3}\right) \sin \left[\pi\left(\frac{1}{2}+\frac{h}{3}\right)\right] \Gamma\left(\frac{1}{2}\right)} \\
& \cdot \int_{C^{\prime}} \tilde{\rho}(v)^{-1 / 6-h / 3} G\left(v,\left(\frac{1}{4}+\frac{h}{3}\right)\right)\left[\frac{f_{3}(v)}{\frac{1}{6}+\frac{h}{3}}+f_{4}(v)\right] d v
\end{aligned}
$$

The behavior of the integral (7.15) for large values of $\mu$ will determine the region of the $\eta, \theta$-plane for which a deformation of the path of integration in the complex $\mu$-plane is admissible. If one inserts the asymptotic expression (1.18) for the function $G_{1}$ into Eq. (7.15), one finds for the integrand

$$
\left|\frac{1-v^{3}}{v^{3}}\right|^{1 / 12} \tilde{\rho}^{1 / 12}\left[4 \tilde{\rho}(v) \frac{1-v^{3 / 2}}{1+v^{3 / 2}}\right]^{-\mu}\left(\frac{f_{3}(v)}{\mu-\frac{1}{12}}+f_{4}(v)\right)
$$

One must now deform the contour $C^{\prime}$ in such a manner that the minimum of $\tilde{\rho}(v)\left(1-v^{\frac{3}{2}}\right) /\left(1+v^{\frac{3}{2}}\right)$ which occurs along it is as large as possible, while $f_{3} /(\mu-1 / 12)+f_{4}$ is bounded. This will give the largest possible range of validity of the transformations below. The choice of this contour depends obviously upon the function $\tilde{\rho}$ and upon the functions $f_{3}$ and $f_{4}$, for a singularity of the function $f_{3} /(\mu-1 / 12)+f_{4}$ in the complex $v$-plane would obviously limit the deformation of the path of integration $C^{\prime}$, but the deformation $C^{\prime}$ may also be limited by the properties of the expression $\tilde{\rho}(v)$.

Rather than attempt a general discussion, we shall introduce at this point special forms for the expression $\tilde{\rho}(\xi)$.

Case a

$$
\tilde{\rho}(\xi)=\text { const. }\left(1-\xi^{3}\right)
$$

hence

$$
\frac{\tilde{\eta}^{3}}{\xi^{3}}\left(1-\xi^{3}\right)=\left(1-\xi^{3}\right) \text { const. }
$$


or

$\theta=$ const.

Case b

$$
\tilde{\rho}(\xi)=\text { const. } \frac{1-\xi^{\frac{3}{2}}}{1+\xi^{\frac{3}{2}}}
$$

hence

$$
\frac{3}{2} \theta+\eta^{\frac{3}{2}}=\text { const. }
$$

In the first case the curve $C$ is a line $\theta=$ const. through the point $\xi_{1}$, in the second case $\tilde{\rho}(\xi)$ is a characteristic with a negative slope. For any other particular case the discussion can be carried out in a similar manner.

Then one has

$$
4 \tilde{\rho}(\xi) \frac{1-\xi^{\frac{3}{2}}}{1+\xi^{\frac{3}{2}}}=\left[2 \text { const. }\left(1-\xi^{\frac{3}{2}}\right)\right]^{2}
$$

for Case a, and

$$
4 \tilde{\rho}(\xi) \frac{1-\xi^{\frac{3}{2}}}{1+\xi^{\frac{3}{2}}}=\left[2 \text { const. } \frac{1-\xi^{\frac{3}{2}}}{1+\xi^{\frac{3}{2}}}\right]^{2}
$$

for Case b.

Now assume that a singularity of $\left(f_{3} /(\mu-1 / 12)+f_{4}\right)$ lies at the point $\xi_{2}$ of the complex $\xi$-plane. Then we choose as contour $C^{\prime}$ in the complex plane a circle for which in Case a

$$
\mid\left(1-\xi^{\frac{3}{2}}|<| 1-\xi_{2}^{\frac{3}{2}} \mid\right.
$$

and in Case b

$$
\left|\frac{1-\xi^{\frac{3}{2}}}{1+\xi^{\frac{3}{2}}}\right|<\left|\frac{1-\xi_{2}^{\frac{3}{2}}}{1+\xi_{2}^{\frac{3}{2}}}\right|
$$

If $\xi_{2}$ lies at a real value of $\xi$ then it coincides with $\xi_{1}$, i.e. it represents the limit of the interval in which $f_{1}, f_{2}$, and $\tilde{\rho}$ are supposed to be analytic. If $\xi_{2}$ lies somewhere in the complex $\xi$-plane, then we can define $\xi_{1}$ such that

in Case a

$$
\left|1-\xi_{1}^{\frac{3}{2}}\right|=\left|1-\xi_{2}^{\frac{3}{2}}\right|
$$

and in Case b

$$
\left|\frac{1-\xi_{1}^{\frac{3}{2}}}{1+\xi_{1}^{\frac{3}{2}}}\right|=\left|\frac{1-\xi_{2}^{\frac{3}{2}}}{1+\xi_{2}^{\frac{3}{2}}}\right|
$$

With the choice of the contour of integration given in Eqs. (7.17) the modulus of the expressions $4 \tilde{\rho}\left(1-v^{\frac{3}{2}}\right) /\left(1+v^{\frac{3}{2}}\right)$ is constant, the argument changes in each case from 0 to $-2 \pi i$.

Thus one obtains finally as estimate for $J(\mu)$

in Case a

$$
|J(\mu)| \leqq M\left[2 \text { const. }\left(1-\xi_{2}^{\frac{3}{3}}\right)\right]^{-2 \mathrm{Re} \mu}
$$

and in Case b

$$
|J(\mu)| \leqq M\left[2 \text { const. } \frac{1-\xi_{2}^{\frac{3}{2}}}{1+\xi_{2}^{\frac{1}{2}}}\right]^{-2 \mathrm{Re} \mu}
$$


With the definition given above for $\xi_{1}$, this can be written in either case as

$$
|J(\mu)| \leqq M\left[4 \tilde{\rho}\left(\xi_{1}\right) \frac{1-\xi_{1}^{\frac{3}{2}}}{1+\xi_{1}^{\frac{3}{2}}}\right]^{-\mathrm{Re} \mu}
$$

After these investigations of the integral $J$, one can determine the region in which a deformation of the path of integration in Eq. (7.11) from the imaginary to the real axis of the complex $\mu$-plane can be carried out. One finds that the integrand of Eq. (7.11) is smaller than

$$
M \frac{G(\xi, \mu) \rho^{\mu}}{\mu B(-\mu) \sin \left[\pi\left(-\mu+\frac{1}{4}\right)\right]}\left[4 \tilde{\rho}\left(\xi_{1}\right) \frac{1-\xi_{1}^{\frac{3}{2}}}{1+\xi_{1}^{\frac{3}{2}}}\right]^{-\mathrm{Re} \mu}
$$

Here one must introduce the asymptotic expressions for $B(-\mu)$ and for $G$; $B(\mu)$ is given by Equation (4.5).

$$
\begin{aligned}
B(-\mu) & =\Gamma\left(\frac{1}{2}\right) \frac{\Gamma(-2 \mu)}{\Gamma\left(-\mu+\frac{11}{12}\right) \Gamma\left(-\mu+\frac{7}{12}\right)} \\
& =\frac{\Gamma\left(\mu+\frac{1}{12}\right) \Gamma\left(\mu+\frac{5}{12}\right) \sin \left[\pi\left(\mu+\frac{1}{12}\right)\right] \sin \left[\pi\left(\mu+\frac{5}{12}\right)\right]}{\sqrt{\pi} \Gamma(1+2 \mu) \sin [\pi(1+2 \mu)]} \\
& =\frac{\Gamma\left(\mu+\frac{1}{12}\right) \Gamma\left(\mu+\frac{5}{12}\right) \sin \left[\pi\left(\mu+\frac{1}{12}\right)\right] \sin \left[\pi\left(\mu+\frac{5}{12}\right)\right]}{\Gamma\left(\mu+\frac{1}{2}\right) \Gamma(\mu+1) 2^{2 \mu} \sin [\pi(1+2 \mu)]} \\
& \sim \frac{1}{\mu} \frac{\sin \left[\pi\left(\mu+\frac{1}{12}\right)\right] \sin \left[\pi\left(\mu+\frac{5}{12}\right)\right]}{2^{2 \mu} \sin [\pi(1+2 \mu)]}
\end{aligned}
$$

Thus one obtains as an estimation of the integrand of (7.11) for large values of $|\mu|$ and suitable contours $C_{n}$

$$
G(\xi, \mu) e^{-\pi \operatorname{Im} \mu}\left(\frac{\tilde{\rho}}{\rho} \frac{1-\xi_{1}^{\frac{3}{2}}}{1+\xi_{1}^{\frac{3}{3}}}\right)^{-\operatorname{Re} \mu}
$$

From here on, the discussion regarding the region of the $\eta, \theta$-plane in which the deformation of the path of integration in the $\mu$-plane is admissible is the same as for $\psi_{\mathrm{I}}$.

In the discussion just given the case was included where a singularity of $f_{3}$ or $f_{4}$ does not lie at a real value of $\xi$. In this case the location of the point $\xi_{1}$ is not evident from the boundary values but arises in the course of the computations. It is remarkable that the location of $\xi_{1}$ depends also upon $\tilde{\rho}(\xi)$. Even if $f_{3}, f_{4}$ and $\tilde{\rho}$ are analytic functions for real values of $\tilde{\rho}$ in the entire supersonic part of curve $C$, the point $\xi_{1}$ from which the construction of the region of convergence shown in Fig. 6 starts will in general not lie at the sonic velocity; it must be expected to lie at some point of the supersonic arc of $C$. Naturally the 
above considerations give only a sufficient condition for the region of convergence; however, the fact that along a line $\xi=$ const. $\psi$ is represented as a power series in $\rho$ will lead to the same conclusion, except perhaps for the exact location of the point $\xi_{1}$.

Now we can carry out the deformation of the path of integration in the complex $\mu$-plane. Let us consider first the poles which are due to the integral $J$.

The function $G_{1}$ in the integral is a hypergeometric function which has a pole if the third parameter is a negative integer. Thus one series of poles will lie at integral and half integral values of $\mu$. Plainly another pole lies at $\mu=1 / 12$. Finally, poles will possibly arise at the zeros of the expression $\left\{1-e^{\left.-2 \pi i_{1}\left(\alpha_{1}+1\right)(1 / 12-\mu)+\alpha_{2}\right]}\right\}$ occurring in front of the integral (7.15).

If one now considers the entire integrand of (7.11) then one finds that the poles at integral and half integral values of $\mu$ are cancelled by zeros of the expression $[B(-\mu)]^{-1}$.

The residue at the point $\mu=1 / 12$ is found to be

$$
\frac{-i G\left(\xi, \frac{1}{12}\right)}{\left(1-e^{-2 i \pi \alpha_{2}}, \frac{1}{6} B\left(-\frac{1}{12}\right) \frac{1}{2}\right.} \int_{C^{\prime}} G_{1}\left(v,-\frac{1}{12}\right) f_{3}(v) d v
$$

Using the definitions of $f_{3}$ (Eq. 7.2) and taking into account that $G_{1}$ satisfies Eq. (1.7), one obtains

$$
i \frac{2}{3} \frac{G\left(\xi, \frac{1}{12}\right)}{B\left(-\frac{1}{12}\right) \sin \frac{\pi}{6}}\left(1-\xi_{1}^{3}\right)^{5 / 6}\left[f_{1}^{\prime}\left(\xi_{1}\right) G_{1}\left(\xi_{1},-\frac{1}{12}\right)-f_{1} G_{1}^{\prime}\left(\xi_{1},-\frac{1}{12}\right)\right]
$$

If the expression $1-e^{-2 \pi i\left[\left\{1+\alpha_{1}\right)(1 / 12-\mu)+\alpha_{2}\right]}$ in the denominator of Eq. (7.15) vanishes, then the integrand of $J$ is a one-valued function of $v$. The values of $\mu$ for which this happens may be denoted by $\mu_{h}^{\prime}$. One has

$$
\mu_{h}^{\prime}=\frac{1}{12}+\frac{\alpha_{2}+h}{1+\alpha_{1}}
$$

where $h$ is an integer.

Since the path of integration $C^{\prime}$ in Eq. (7.15) is a closed curve, along which one proceeds in the counterclockwise direction, one finds in this case as value of the integral $-2 \pi i$ times the residue of the integrand at $v=1$. Naturally, if $h$ in Eq. (7.20) is sufficiently small, then the integrand will be regular at $v=1$, i.e. not all the points $\mu_{h}^{\prime}$ given in Eq. (7.20) are actually poles. As residue of the pole of the integral of $E_{4}+E_{6}$ (Eq. 7.11) at the point $\mu=\mu_{h}^{\prime}$ one finds

$$
\begin{aligned}
& \frac{-i G\left(\xi, \mu_{h}^{\prime}\right)}{2 \mu_{h}^{\prime} B\left(-\mu_{h}^{\prime}\right) \sin \left(-\mu_{h}^{\prime}+\frac{1}{4}\right)} \rho^{-1 / 12+\mu^{\prime} h} \frac{1}{\alpha_{1}+1} \\
& \cdot\left\{\text { Residue at } v=1 \text { of } \tilde{\rho}(v)^{-1 / 12-\mu_{h}^{\prime} h} G_{1}\left(v,-\mu_{h}^{\prime}\right)\left[\frac{f_{3}(v)}{\mu_{h}^{\prime}-\frac{1}{12}}+f_{4}(v)\right]\right\}
\end{aligned}
$$


Furthermore poles are caused by the factor $2 \mu B(-\mu) \sin \left[\pi\left(-\mu+\frac{1}{4}\right)\right]$ occurring in Eq. (7.12). Since the calculation is very similar to investigations carried out previously, we give only the results. Poles occur at the points $\mu=\frac{1}{4}+h / 3$, i.e. at exactly those points which correspond to the particular solutions of the first family. The residues are found as

$$
\frac{-i G\left(\xi, \frac{1}{4}+\frac{h}{3}\right) \sqrt{\pi} \rho^{1 / 6+h / 3} J\left(\frac{1}{4}+\frac{h}{3}\right) \Gamma\left(\frac{1}{2}+\frac{2 h}{3}\right)}{\Gamma\left(\frac{1}{3}+\frac{h}{3}\right) \Gamma\left(\frac{2}{3}+\frac{h}{3}\right)} \frac{4}{3 \pi} \cos \frac{\pi h}{3}
$$

Thus, finally, by combining the Eqs. (7.19), (7.21) and (7.22) and by inserting into Eq. (7.22) the expression (7.15a) one obtains

$$
\begin{aligned}
& E_{4}+E_{6}=\sum_{h=0}^{\infty}-G\left(\xi, \frac{1}{4}+\frac{h}{3}\right) \rho^{1 / 6+h / 3} \frac{\Gamma\left(\frac{7}{6}+\frac{h}{3}\right) \Gamma\left(\frac{5}{6}+\frac{h}{3}\right)}{\Gamma\left(\frac{1}{3}+\frac{h}{3}\right) \Gamma\left(\frac{2}{3}+\frac{h}{3}\right)} \frac{4}{3} \\
& \cdot\left\{1-e^{\left.-2 i \pi_{\{}\left(1+\alpha_{1}\right)(-1 / 6-h / 3)+\alpha_{2}\right\}}\right\}^{-1} \int_{C^{\prime}} \tilde{\rho}(v)^{-1 / 6-h / 3} G\left(v, \frac{1}{4}+\frac{h}{3}\right)\left[\frac{f_{3}(v)}{\frac{1}{6}+\frac{h}{3}}+f_{4}(v)\right] d v \\
& +\frac{2 \pi G\left(\xi, \frac{1}{12}\right)}{3 B\left(-\frac{1}{12}\right) \sin \frac{\pi}{6}}\left(1-\xi_{1}^{3}\right)^{5 / 6}\left[f_{1}^{\prime}\left(\xi_{1}\right) G_{1}\left(\xi_{1},-\frac{1}{12}\right)-f_{1}\left(\xi_{1}\right) G_{1}^{\prime}\left(\xi_{1},-\frac{1}{12}\right)\right] \\
& -\sum_{\mu^{\prime} h} \frac{\pi G\left(\xi, \mu_{h}^{\prime}\right)}{2 \mu_{h}^{\prime} B\left(-\mu_{h}^{\prime}\right) \sin \left[\pi\left(-\mu_{h}^{\prime}+\frac{1}{4}\right)\right]} \rho^{-1 / 12+\mu^{\prime} h} \\
& \frac{1}{\alpha_{1}+1}\left[\text { Residues at } v=1 \text { of } \tilde{\rho}(v)^{-1 / 12-\mu^{\prime} h} G_{1}\left(v,-\mu_{h}^{\prime}\right)\left[\frac{f_{3}(v)}{\mu_{h}^{\prime}-\frac{1}{12}}+f_{4}(v)\right]\right]
\end{aligned}
$$

By means of the last equation and Eq. (7.11), one can then determine $\psi_{\mathrm{II}}$ (Eq. 7.8). In the expression thus arising we consider first the term

$$
\begin{aligned}
\frac{3}{\pi} G\left(\xi, \frac{1}{12}\right)\left(1-\xi_{1}^{3}\right)^{5 / 6} \frac{2 \pi}{3}\left[f_{1}^{\prime}\left(\xi_{1}\right)\left(\frac{G_{1}\left(\xi_{1},+\frac{1}{12}\right)}{B\left(\frac{1}{12}\right) \sin \frac{\pi}{3}}+\frac{G_{1}\left(\xi_{1},-\frac{1}{12}\right)}{B\left(-\frac{1}{12}\right) \sin \frac{\pi}{6}}\right)\right. \\
-f_{1}\left(\xi_{1}\right)\left(\frac{G_{1}^{\prime}\left(\xi_{1}, \frac{1}{12}\right)}{B\left(\frac{1}{12}\right) \sin \frac{\pi}{3}}+\frac{G_{1}^{\prime}\left(\xi_{1},-\frac{1}{12}\right)}{\left.\left.B\left(-\frac{1}{12}\right) \sin \frac{\pi}{6}\right)\right]}\right.
\end{aligned}
$$

Inserting Eqs. (1.17) and (4.5) into Eq. (1.10), one obtains

$$
G\left(\xi, \frac{1}{12}\right)=B\left(\frac{1}{12}\right) \sin \frac{\pi}{3} G_{1}\left(\xi,-\frac{1}{12}\right)+B\left(-\frac{1}{12}\right) \sin \frac{\pi}{6} G_{1}\left(\xi, \frac{1}{12}\right)
$$


Thus the last expression can be written as

$$
\frac{2 G\left(\xi, \frac{1}{12}\right)\left(1-\xi_{1}^{3}\right)^{5 / 6}}{B\left(\frac{1}{12}\right) B\left(-\frac{1}{12}\right) \sin \frac{\pi}{3} \sin \frac{\pi}{6}}\left[f_{1}^{\prime}\left(\xi_{1}\right) G\left(\xi_{1}, \frac{1}{12}\right)-f_{1}\left(\xi_{1}\right) G^{\prime}\left(\xi_{1}, \frac{1}{12}\right)\right]
$$

This term occurs in $\psi_{\mathrm{I}}$ with the opposite sign. We give immediately the final expression for the solution $\psi=\psi_{\mathrm{I}}+\psi_{\mathrm{II}}$ where $\psi_{\mathrm{I}}$ is found in Eq. (7.5)

$$
\begin{aligned}
& \psi=\frac{4}{\pi} \sum_{h=0}^{\infty}-G\left(\xi, \frac{1}{4}+\frac{h}{3}\right) \rho^{1 / 6+h / 3} \frac{\Gamma\left(\frac{h}{3}+\frac{7}{6}\right) \Gamma\left(\frac{h}{3}+\frac{5}{6}\right)}{\Gamma\left(\frac{h}{3}+\frac{1}{3}\right) \Gamma\left(\frac{h}{3}+\frac{2}{3}\right)} \\
& \cdot\left\{\int_{-\infty}^{\xi_{1}} \tilde{\rho}(v)^{-1 / 6-h / 3} G\left(v, \frac{1}{4}+\frac{h}{3}\right)\left[\frac{f_{3}(v)}{\frac{h}{3}+\frac{1}{6}}+f_{4}(v)\right] d v\right. \\
& +\left[1-e^{-2 i \pi_{[}\left(1+\alpha_{1}\right)(-1 / 6-h / 3)+\alpha_{2}}\right]^{-1} \\
& \left.\cdot \int_{C}, \tilde{\rho}(v)^{-1 / 6-h / 3} G\left(v, \frac{1}{4}+\frac{h}{3}\right)\left[\frac{f_{3}(v)}{\frac{h}{3}+\frac{1}{6}}+f_{4}(v)\right] d v\right\} \\
& -3 \sum_{\mu^{\prime} h} \frac{G\left(\xi, \mu_{h}^{\prime}\right) \rho^{-1 / 12+\mu^{\prime} h}}{2 \mu_{h}^{\prime} B\left(-\mu_{h}^{\prime}\right) \sin \left[\pi\left(-\mu_{h}^{\prime}+\frac{1}{4}\right)\right]} \\
& \text { - } \frac{1}{\alpha_{1}+1}[\text { Residues at } v=1 \text { of } \\
& \left.\tilde{\rho}(v)^{-1 / 12-\mu^{\prime} h} G_{1}\left(v,-\mu_{h}^{\prime}\right)\left[\frac{f_{3}(v)}{\mu_{h}^{\prime}-\frac{1}{12}}+f_{4}(v)\right]\right]
\end{aligned}
$$

Here $\alpha_{1}$ and $\alpha_{2}$ are defined in Eq. (7.13), $\mu_{h}^{\prime}$ in (7.20). The choice of $\xi_{1}$ is explained after Eqs. (7.17).

While the first sum is a superposition of particular solutions of the first family, the second sum introduces a new family of particular solutions. But their presence should not be surprising. Because of the nature of the boundary conditions given along $C$ in the vicinity of $\xi=1$, singularities may propagate along this line. The character of these singularities can be determined completely from the series development of the functions $\tilde{\rho}, f_{1}$, and $f_{2}$ at the point $\xi=1$. The particular solutions of the first system are not suitable for representing the propagation of such singularities, for they are determined by the requirement that all derivatives exist along $\xi=1$. Now a detailed study of the second sum would show that it gives exactly that superposition of particular solutions (2.2) which is required in order to represent the singularities propagating along $\xi=1$.

Although it would be possible to determine such a superposition of particular 
solutions by inspection, the present method of evaluating the residues appears to be preferable because of its conciseness and because of the possibility of settling the question of convergence at the same time. As was mentioned previously, the first integral in Eq. (7.24) would diverge if its upper limit were $v=1$. This was the reason for the separate treatment of $\psi_{\mathrm{I}}$ and $\psi_{\mathrm{II}}$. To overcome this difficulty a redefinition of this integral was carried out in Eq. (7.15). The appearance of the second sum in Eq. (7.24) as well as the form of the second term in the first sum is a consequence hereof.

One will note that Equation (7.24) remains unchanged if the value of $\xi_{1}$ is changed, e.g. if $\xi_{1}$ is chosen closer to one. However the value of $\xi_{1}$ is of great importance for the determination of the region of convergence for the expression (7.24).

\section{Concluding Remarks}

The present paper is concerned with the development of a solution of TRIcomr's equation in the vicinity of a certain point on the line $\eta=0$. A family of particular solutions which appears suitable for this purpose is readily found; they fulfill the condition that $\psi$ and all of its derivatives exist along the characteristics going through this point. The question remains whether this system is sufficiently general.

Eq. (7.24) gives the desired representation. If the function $\psi$ is of such a nature that all derivatives exist along the characteristic $\xi=1$, then a representation in terms of the particular solutions mentioned above is always possible. Otherwise further particular solutions, which put into evidence the singularities which will then propagate along $\xi=1$, will be present.

The main application of these investigations lies in the theory of transonic flow. In Reference 2 has been investigated how the flow field over a given body with a free stream Mach number in the vicinity of one can be developed with respect to the deviation of the free stream Mach number from one. For this purpose the representation of a general solution in terms of a complete system of particular solutions was needed. Since at the time when Reference 2 was written the completeness of the first family of particular solution was not established, these investigations use the second family, for which the completeness is clear immediately. But the representation thus obtained is somewhat unsatisfactory. The conditions imposed on the particular solutions along $\xi=1$ are somewhat artificial, and consequently a representation of a given solution in terms of the second family of particular solutions does not at all display the behavior of the original expression along the line $\xi=1$ and at the origin. The present paper establishes the equivalence of the first and second families of particular solutions and thus overcomes this difficulty.

The fact that in Eq. (7.24) a function $\psi$ is expressed in the form of a power series development with respect to $\rho$ is of importance for investigations where the behavior of the solution in the vicinity of point 0 is required. E.g., it would 
be possible with the results of the present paper to make the investigations of Reference 2 more definite and to extend them to approximations of a higher order. Another example where the present results are important is shown in Reference 11.

In solving boundary value problems it will frequently be impossible to obtain the solution immediately in terms of the particular solutions of the first family. Here Eq. (7.24) allows one to calculate this representation after the boundary value problem has been solved by means of another method. In this regard Eq. (7.24) can be compared to CAUCHY's formula for the value of an analytic function and of its derivatives.

\begin{abstract}
For some problems of transonic flow, it is desirable to represent solutions of TRICOMI's equation by a superposition of particular solutions. Suitable particular solutions are found easily, but it is far from obvious how to select among them a system allowing the representation of an arbitrary solution. A first family of particular solutions is obtained by imposing conditions originating from the physical properties of the solutions. However, nothing is then known about the completeness of the system; in fact there are indications that such a representation is not generally applicable.

A second family of particular solutions is defined by a means of a somewhat artificial boundary condition; here the particular solutions are determined by an eigenvalue problem, and the completeness of the system follows from known theorems. The representation of an arbitrary function of one variable by means of the eigenfunctions of this system requires a limiting process similar to the one which leads from a Fourier series to a Fourier integral. With the formula thus obtained it is possible to solve a certain inhomogeneous boundary value problem formulated in such a manner that its solution represents an arbitrary solution of TRICOMI's equation. This solution appears first in a rather complex form, but by deforming the path of integration in some integrals occurring there, the solution can finally be expressed in the desired form, i.e., as a superposition of particular solutions of the first family.

Certain restrictive conditions, mainly concerning the region of convergence of this representation, appear automatically in the course of these transformations.
\end{abstract}




\section{REFERENCES}

1. Tricomi, F., Sulle equazioni lineari alle derivate pariali do $2^{\circ}$ ordine di tipo misto, Atti della R. Accademia Nazionale dei Lincei 1923, Serie Quinta Memoria della Classe di Science Fisiche, Matematiche e Naturali, Volume XIV, p. 134. (English Translation by Brown University).

2. Guderley, Gottrried, Two-dimensional Flow Patterns with a Free Stream Mach Number Close to One, AF Technical Report No. 6343, May 1951.

3. Guderley, GotTfried, Singularities at the Sonic Velocity, Headquarters Air Materiel Command, Wright-Patterson Air Force Base, Dayton, Ohio, Tech. Report F-TR-1171-ND.

4. Guderlex, Gottrried, The Wall Pressure Distribution in a Choked Tunnel, Wright Air Development Center, WADC Tech. Report 53-509 (1953).

5. Langer, R. E., On Asymptotic Solutions of Ordinary Differential Equations with an Application to Bessel Functions of Large Order, Trans. Amer. Math. Soc., 1931, pp. 23 to 64.

6. Guderley, Gotrfried, The Flow over a Flat Plate with a small Angle of Attack at Mach Number 1, Journal of the Aeronautical Sciences 1954, pp. 261-274.

7. Guderley, GotTfried, The Flat Plate with an Angle of Attack in a choked Wind Tunnel, to appear in the Journal of the Aeronautical Sciences.

8. HAMEL, GeorG, Integralgleichungen, Berlin, Springer Verlag 1937, pp. 121-122.

9. Guderley, Gottrried, A Formula for the Normalization Constant in Eigenvalue Problems, Quarterly of Applied Mathematics, Vol. X, 1952, p. 176.

10. Copson, E. T., An Introduction to the Theory of a Complex Variable, Oxford University Press.

11. Guderley, Gottrried, On Transonic Airfoil Theory, Journal of the Aeronautical Sciences. 